\title{
1 Inhibitors of VPS34 and lipid metabolism suppress SARS-CoV-2 replication
}

2 Jesus A. Silvas, 1,3, Alexander S. Jureka ${ }^{1,3}$, Anthony M. Nicolini' ${ }^{2}$, Stacie A. Chvatal ${ }^{2}$, and

3 Christopher F. Basler ${ }^{1 *}$

$4{ }^{1}$ Center for Microbial Pathogenesis, Institute for Biomedical Sciences, Georgia State University,

5 Atlanta, GA, 30303

$6 \quad 2$ Axion BioSystems, Inc., Atlanta, GA 30309

$7 \quad{ }^{3}$ Equal contribution

$8 *$ Corresponding Author

9 Christopher F. Basler PhD

10 Center for Microbial Pathogenesis

11 Institute for Biomedical Sciences

12 Georgia State University

13 Atlanta, GA 30307

14 (404) 413-3651

15 cbasler@gsu.edu 


\section{ABSTRACT}

26 Therapeutics targeting replication of SARS coronavirus 2 (SARS-CoV-2) are urgently needed.

27 Coronaviruses rely on host membranes for entry, establishment of replication centers and egress.

28 Compounds targeting cellular membrane biology and lipid biosynthetic pathways have

29 previously shown promise as antivirals and are actively being pursued as treatments for other

30 conditions. Here, we tested small molecule inhibitors that target membrane dynamics or lipid

31 metabolism. Included were inhibitors of the PI3 kinase VPS34, which functions in autophagy,

32 endocytosis and other processes; Orlistat, an inhibitor of lipases and fatty acid synthetase, is

33 approved by the FDA as a treatment for obesity; and Triacsin C which inhibits long chain fatty

34 acyl-CoA synthetases. VPS34 inhibitors, Orlistat and Triacsin C inhibited virus growth in Vero

35 E6 cells and in the human airway epithelial cell line Calu-3, acting at a post-entry step in the

36 virus replication cycle. Of these the VPS34 inhibitors exhibit the most potent activity. 


\section{INTRODUCTION}

SARS-CoV-2, a member of the Betacoronavirus genus, is an enveloped positive-sense,

48 RNA virus responsible for a current pandemic ${ }^{1}$. Because of its profound impact on society and

49 human health there is an urgent need to understand SARS-CoV-2 replication requirements and to

50 identify therapeutic strategies ${ }^{2}$. Repurposing drugs developed for other purposes may provide a

51 shortcut to therapeutic development ${ }^{3-6}$. The use of compounds known to target specific host

52 factors may also elucidate key pathways needed for virus replication.

Coronavirus $(\mathrm{CoV})$ replication involves multiple critical interactions with host cell

54 membranes, including during viral entry and virus release $e^{2,7-9}$. In addition, one of the most

55 striking features of $\mathrm{CoV}$ infection is the establishment of replication organelles that consist of

56 double membrane vesicles (DMV), double-membrane spherules (DMSs) and convoluted

57 membranes $(\mathrm{CM})$ with DMVs serving as the main site of viral RNA synthesis ${ }^{10}$. The origin of

58 these membrane organelles in beta-coronavirus infection remains incompletely understood. The

59 membrane structures colocalize with LC3, a protein with well-known functions in autophagy ${ }^{7,11}$.

60 In murine embryonic stem cell lines, autophagy was found to be critical for DMV formation and

61 replication of the beta-coronavirus mouse hepatitis virus ${ }^{7}$. However, studies in bone marrow

62 derived macrophages or primary mouse embryonic fibroblasts lacking ATG5 indicated that

63 autophagy is not essential for DMV formation or MHV replication ${ }^{11}$. An alternate model

64 indicates that beta coronaviruses usurp vesicles known as EDEMosomes, which associate with

65 non-lipidated LC3 and normally function to regulate ER-associated degradation (ERAD), to

66 provide membranes for replication ${ }^{8}$.

67 Many enveloped, positive-sense RNA viruses that replicate in double membrane

68 compartments have been demonstrated to be sensitive to inhibitors of various aspects of 
70 that plays roles in autophagy, endosomal trafficking, and other aspects of membrane biology has

71 been implicated in the replication of hepatitis $\mathrm{C}$ virus (HCV) and tombusvirus (TBSV) ${ }^{12,13}$. The

72 compound Triacsin C, which inhibits an enzyme upstream of triglyceride synthesis, long chain

73 fatty acyl CoA, impairs the growth of several viruses that require for replication lipid droplets,

74 organelles that serve as storage sites for neutral lipids such as triacylglycerol ${ }^{14-16}$. Downstream of

75 long chain fatty acyl CoA in the synthesis of triglycerides are diacylglycerol acyltransferases 1

76 and 2 (DGAT1 and DGAT2). Inhibition of these enzymes inhibits HCV and rotavirus

77 replication. More general inhibitors of fatty acid synthetase such as Orlistat, also decrease

78 replication of several different viruses ${ }^{17-20}$.

79 Here we asked whether SARS-CoV-2 is susceptible to modulators of lipid metabolism by

80 assessing the sensitivity of the virus in Vero E6 and Calu-3 cells to VPS34 inhibitors, Triacsin C,

81 inhibitors of DGATs and Orlistat, an inhibitor of FASN ${ }^{21}$. We find that two inhibitors of VPS34

82 potently inhibited SARS-CoV-2 replication, whereas an FDA-approved inhibitor of a different

83 class of PI3K had minimal effect on replication. Targeting FASN and de novo synthesis of

84 triacylglycerol, diacylglycerol and cholesterol esters each impairs SARS-CoV-2 replication

85 whereas inhibition of DGATs was not effective. We also identified that each inhibitor exhibits

86 antiviral effects post-entry and that they perturb the structure of viral replication centers. Taken

87 together, the data presented here implicates specific lipid metabolism pathways in SARS-CoV-2

88 replication and suggests that these pathways are promising therapeutic targets. 


\section{$91 \quad$ Virus and cell lines}

93 were maintained in DMEM (Corning) supplemented with 10\% heat inactivated fetal bovine

94 serum (FBS; GIBCO). Cells were kept in a $37^{\circ} \mathrm{C}, 5 \% \mathrm{CO}_{2}$ incubator without antibiotics or

95 antimycotics. SARS-CoV-2, strain USA_WA1/2020, was obtained from the World Reference

96 Collection for Emerging Viruses and Arboviruses at the University of Texas Medical Branch-

97 Galveston.

Virus Propagation and Plaque Assays

A lyophilized ampule of SARS-CoV-2 was initially resuspended in DMEM

101 with an adsorption period of 1 hour at 37C and shaking every 15 minutes. Cells were observed

102 for cytopathic effect (CPE) every 24 hours. Stock SARS-CoV-2 virus was harvested at 72 hours

103 post infection (h.p.i) and supernatants were collected, clarified, aliquoted, and stored at $-80^{\circ} \mathrm{C}$.

For plaque assays, Vero E6 cells were seeded onto a 24-well plate 24 hours before

105 infection. 100ul of SARS-CoV-2 serial dilutions were added, adsorbed for 1 hour at 37C with

106 shaking at 15-minute intervals. After the absorption period, $1 \mathrm{~mL}$ of $0.6 \%$ microcrystalline

107 cellulose (MCC; Sigma 435244) in serum-free DMEM was added. To stain plaque assays MCC

108 was removed by aspiration, and 10\% neutral buffered formalin (NBF) added for one hour at

109 room temp and then removed. Monolayers were then washed with water and stained with $0.4 \%$

110 crystal violet. Plaques were quantified and recorded as plaque forming units (PFU)/mL.

\section{Confocal microscopy}


For confocal microscopy analysis, all cell lines were pre-seeded 24 hours before infection

113 onto glass coverslips and infected with SARS-CoV-2 at a multiplicity of infection (MOI) of 1. At

11424 hours post-infection (h.p.i.) supernatant was removed, and samples fixed with 10\% NBF for 1

115 hour at room temperature followed by PBS wash and permeabilized with sterile filtered $0.1 \%$

116 Saponin in PBS. Cells were blocked with 0.1\% Saponin in Fluorescent Blocker (ThermoFisher)

117 for 1 hour at RT. Primary antibodies were added and incubated overnight at 4C. AlexaFluor488, 118 594, and 647 conjugated secondary antibodies were used and nuclei stained with DAPI. Samples

119 were imaged on Zeiss LSM800 Confocal with Super Resolution AiryScan. Images were 120 rendered in ZenBlue or Imaris Viewer 9.0.

\section{Maestro Z Impedance Experiments}

Prior to cell plating, CytoView-Z 96-well electrode plates (Axion BioSystems, Atlanta,

$123 \mathrm{GA}$ ) were coated with $5 \mu \mathrm{g} / \mathrm{mL}$ human fibronectin (Corning) for $1 \mathrm{hr}$ at 37C. After coating,

124 fibronectin was removed and $100 \mu \mathrm{L}$ of DMEM/10\% FBS was added to each well. The plate was

125 then docked into the Maestro $\mathrm{Z}$ instrument to measure impedance electrode baseline. Vero E6

126 cells were then plated to confluency $(\sim 75,000$ cells/well $)$ in the coated CytoView-Z plates and

127 left at room temperature for 1 hour to ensure even coverage of the well. Plates containing Vero

128 E6 cells were then docked into the Maestro $\mathrm{Z}$ for 24 hours at $37^{\circ} \mathrm{C} / 5 \% \mathrm{CO}_{2}$ to allow the cells to

129 attach and the monolayer to stabilize, as measured by resistance, a component of impedance. The

130 Maestro $\mathrm{Z}$ was used to monitor the resistance of the monolayer as it formed, very similar to 131 transepithelial electrical resistance $(\text { TEER })^{22}$. In this study, resistance was measured at $10 \mathrm{kHz}$,

132 which reflects both cell coverage over the electrode and strength of the barrier formed by the cell

133 monolayer. For compound treatments, media was removed from wells of the CytoView-Z plates

134 and $195 \mu \mathrm{L}$ of pre-warmed DMEM/2\% FBS was added with the indicated concentration of 
135 compound. Infections with SARS-CoV-2 at an MOI of 0.01 were carried out by directly adding

$1365 \mu \mathrm{L}$ of virus to each well. Plates were then docked within the Maestro $\mathrm{Z}$ and resistance

137 measurements were continuously recorded for 48-72 hours post-infection. All plates contained

138 media only, full lysis, uninfected, and SARS-CoV-2 infected controls. For calculation of percent

139 inhibition, raw resistance values for each well were normalized to the value at 1 hour post-

140 infection within the Axis $\mathrm{Z}$ software, and percent inhibition was calculated with the following

141 formula: Percent Inhibition $=100 *(1-(1-$ average of treated cells $) /(1$-average of infected

142 control)). Median time to death calculations were performed by fitting the Boltzmann sigmoid

143 equation to raw kinetic resistance data in Graphpad Prism. Fifty percent maximum velocity

144 (V50) values obtained from the Boltzmann sigmoid fits were used to determine median time to

145 death for each MOI.

\section{Cell viability assay}

VeroE6 or Calu-3 cells were seeded in 96-well black walled microplates and incubated

148 overnight. Cells were then treated with compounds and CellTox Green Dye (Promega) to

149 monitor compound cytotoxicity. Fluorescence (Excitation: 485nm, Emission: 520nm) was

150 measured every 24 hours post treatment for 3 days. Percent viability was determined using the

151 minimum fluorescence obtained from media only cells and the maximum value obtained by cells

152 lysed with $1 \%$ Triton-X.

\section{Labeling of nascent viral RNA}

VeroE6 cells were seeded onto glass coverslips and incubated overnight at 37C. Cells

155 were then infected with SARS-CoV-2 at an MOI of 3. At 24 h.p.i. cells were treated with $1 \mu \mathrm{M}$

156 of Actinomycin D (Sigma) for 1 hour. Nascent RNA was labeled using Click-iT ${ }^{\text {TM }}$ RNA 
157 Alexa Fluor 594 Imaging Kit (ThermoFisher). Cells were then processed for confocal 158 analysis.

159 Compounds

161 Orlistat (\#10005426) were purchased from Cayman Chemical (Ann Arbor,

162 Michigan). Remdesivir was purchased from Target Molecule Corp. (T7766, Boston, 163 Massachusetts). T863 (\#SML0539) and PF06424439 (\#PZ0233) were purchased from Sigma164 Aldrich (St. Louis, Missouri). All chemicals were resuspended in dimethylsulfoxide 165 (DMSO). ${ }^{23}$

166 RESULTS

167 Development of 96-well format assay to measure SARS-CoV-2 cytopathic effects

SARS-CoV-2 induces significant cytopathic effects in infected Vero E6 cells. Based on this property, we standardized a 96-well format assay that provides continuous real-time, label-

170 free monitoring of the integrity of cell monolayers, thereby providing assessment of virus growth

171 through decreased cell viability. This assay was standardized using the Maestro Z platform

172 (Axion BioSystems, Atlanta, GA), an instrument that uses 96-well plates containing electrodes in

173 each well (CytoView-Z plates). The electrodes measure electrical impedance across the cell 174 monolayer every minute throughout the course of the experiment. As SARS-CoV-2 replication 175 damages the cell monolayer, impedance measurements decrease over time, providing a detailed 176 assessment of infection kinetics. 
at multiple MOIs (10 to 0.0001 ) and resistance measurements were acquired for 72 hours postinfection. As shown in Figure 1A, the progression of infection at each MOI was clearly distinct. A decrease in resistance could be observed as early as 18-20 h.p.i. at an MOI of 10 and 1, and as late as 56 h.p.i. at an MOI of 0.0001 . Depending on MOI, signals reached their nadirs between 32 to 72 h.p.i. To correlate with a decrease in resistance, the raw kinetic data was used to determine the median time to cell death for each MOI (Figure 1B). Based on its desirable kinetics, the MOI of 0.01 was chosen for the screening of compounds for antiviral activities. granted emergency use authorization (EUA) for the treatment of COVID-19 ${ }^{24,25}$. Vero E6 cells were seeded on a CytoView-Z plate, incubated overnight to allow cells to stabilize, pretreated with 6-fold dilutions of Remdesivir for 1 hour and infected with SARS-CoV-2. Resistance measurements were recorded for 48 h.p.i. (Figure 1C). In agreement with previous studies, we determined an 50\% inhibitory concentration (IC50) for Remdesivir of $1.54 \mu \mathrm{M}$ (Figure 1D) ${ }^{24}$.

193 Taken together, these data validate the impedance-based assay described as a tool for screening of potential SARS-CoV-2 therapeutics.

\section{Inhibitors of VPS34 activity impair SARS-CoV-2 growth}

VPS34 is a multifunctional protein involved in autophagy and membrane trafficking.

197 Since coronaviruses induce formation of double membrane vesicles for replication, we wanted to 198 determine if VPS34 activity was essential for SARS-CoV-2 replication. Therefore, we tested two 199 well characterized VPS34 inhibitors IN-1 (referred as VPS34-IN1 below) and PIK-III over a 10200 point dose response in the resistance assay ${ }^{26}$. The compounds were added to pre-plated Vero E6 
202 induced rapid cytotoxicity at $50 \mu \mathrm{M}$ and $16.67 \mu \mathrm{M}$ as indicated by a rapid decrease in resistance

203 measurements between 1 and 20 h.p.i. (Figure 2A and 2C). However, at concentrations of 5.56

$204 \mu \mathrm{M}$ and below, the integrity of the monolayer was preserved relative to the mock-treated control

205 indicating an antiviral effect and an absence of cytotoxicity. Calculations based on normalized

206 resistance measurements at 48 h.p.i for non-toxic doses yielded IC50s of 0.29uM for VPS34-IN1

207 and 0.202uM for PIK-III (Figure 2B and 2D, respectively). Additionally, IC90s of $2.52 \mu \mathrm{M}$

208 (VPS34-IN1) and $1.81 \mu \mathrm{M}$ (PIK-III) were also calculated. These data suggest that the VPS34

209 kinase plays a significant role in SARS-CoV-2 replication and is a potential target for therapeutic

210 intervention.

\section{Inhibition of fatty acid metabolism inhibits SARS-CoV-2 replication}

Fatty acid metabolism leads to production of triglycerides, phospholipids and other

213 molecules ${ }^{27}$. Elongation of the phospholipid membranes can be aided by channeling fatty acid

214 into phospholipid synthesis ${ }^{28}$. Modulation of fatty acid metabolism has been shown to impact

215 several viruses such as dengue virus, hepatitis $\mathrm{C}$ virus, and Old World alphaviruses ${ }^{18,29,30}$. Two

216 well-described compounds that inhibit fatty acid metabolism are Orlistat and Triacsin C, both of

217 which have been shown to have antiviral activity ${ }^{19,}{ }^{30}$. Orlistat is an FDA-approved drug that

218 inhibits lipases and also fatty acid synthase (FASN), and Triacsin C inhibits long chain Acyl-

219 CoA synthetases. To test these against SARS-CoV-2, VeroE6 cells were pre-seeded onto a 220 CytoView-Z plate, allowed to stabilize and then pre-treated with Triacsin C or Orlistat for 1 hour 221 before infection with SARS-CoV-2 at an MOI of 0.01. Based on the toxicity window of 1-20

222 h.p.t. determined with the VPS34 inhibitors, neither Triacsin C nor Orlistat induced early 223 cytotoxic effects, even at the highest concentrations of 50uM and 500uM, respectively (Figure

224 3A and 3C). Both compounds exhibited inhibition at the higher concentrations tested, although 
225 complete inhibition was not achieved even with $500 \mu \mathrm{M}$ of Orlistat. Based on the data we

226 extrapolated an IC50 of 422.3uM for Orlistat and calculated an IC50 of 19.5uM for Triacsin C

227 (Figure 3B and 3D). Viruses such as $\mathrm{HCV}$ and rotavirus that are sensitive to inhibition by

228 Triacsin $\mathrm{C}$ are also impaired by inhibitors of DGATs ${ }^{14,31}$. Therefore, we tested the effects of

229 DGAT1 and DGAT2 inhibitors T863 and PF0642443932, 33. Neither compound displayed any

230 inhibitory activity (Supplemental Figure 1). This data suggests that metabolism of fatty acids

231 plays an important role in SARS-CoV-2 infection.

232 VPS34 inhibitors exhibit potent attenuation of SARS-CoV-2 early and late in its replication

233 cycle

234 Next, time-of-addition studies were performed. We sought to determine how long the

235 addition of VPS34-IN1, PIK-III, Orlistat, or Triacsin C could be postponed before activity was

236 lost. Additionally, this would identify if the anti-viral activity of each compound impacted a pre-

237 or post- viral entry step. As indicated in Figure 4A, 4 conditions were tested 1) single treatment

2381 hour prior to viral infection, with compound removed just prior to infection; 2) 1 hour pre-

239 treatment with continuous dosing; 3) dosing at 2 h.p.i.; and 4) dosing at 4 h.p.i.. VeroE6 cells

240 were pre-seeded onto a CytoView-Z plate and allowed to stabilize, compounds were added, and

241 resistance was monitored for 48 hours after infection. Percent inhibition was calculated based on

242 resistance values at 48 h.p.i. We observed that a single $5 \mu \mathrm{M}$ treatment of VPS34-IN1 or PIK-III

243 inhibited SARS-CoV-2 replication (Figure 4B). Additionally, inhibition was observed even

244 when added after 4 h.p.i. In contrast, removal of Orlistat or Triacsin C before infection,

245 eliminated their efficacy. Maintenance throughout the experiment was inhibitory, as was addition

246 at 2 or 4 hours post infection. Interestingly, delayed treatment with Triacsin $\mathrm{C}$ at $50 \mu \mathrm{M}$ exhibited

247 greater anti-viral activity that initiating the treatment one hour prior to infection. Altogether, 
248 these data demonstrate activity of the VPS34 inhibitors at both early and late, post-entry time

249 points and indicate that the effects of Orlistat and Triacsin C are likely post-entry.

\section{Attenuation of VPS34 kinase activity and fatty acid metabolism inhibit SARS-CoV-2}

\section{1}

252

253

254

255

256

257

258

259

260

261

262

263

264

265

266

267

268

269

270

\section{in a human airway epithelial cell line}

We proceeded to investigate if the inhibitors were effective in the human lung carcinoma cell line, Calu-3, by directly measuring production of infectious virus and cytotoxicity. That this cell line is derived from the human airway and is highly susceptible to infection has established it as a standard for infection studies with SARS-CoV-1, MERS-CoV and SARS-CoV-2 ${ }^{34,35}$. Calu3 cells were plated onto 96-well plates and allowed to reach 95\% confluency. Cells were then pre-treated with a range of concentrations of VPS34-IN1, PIK-III, Triacsin C, Orlistat, DMSO, or mock treated with media alone for 1 hour then infected with SARS-CoV-2 at an MOI of 0.01. Supernatants were collected at 48 h.p.i. and titered on VeroE6 cells by plaque assay. In parallel, to determine cytotoxicity of these compounds, Calu-3 cells were seeded onto 96-well black walled 96-well plates, allowed to reach 95\% confluency and treated with VPS34-IN1, PIK-III, Triacsin C, Orlistat, DMSO, or mock treated with media alone. CellTox Green was added at the time of dosing and fluorescence measured at 48 h.p.i. in order to assess cytotoxicity. Each of the compounds inhibited production of infectious virus, as measured by plaque assay on Vero E6 cells Figure 5A, C, E, and G). In contrast to VeroE6 cells, no cytotoxicity was observed even at the highest dose for each compound in Calu-3 cells. We observed IC50s of $0.55 \mu \mathrm{M}$ (VPS34IN1), $0.12 \mu \mathrm{M}$ (PIK-III), 21.25 $\mu \mathrm{M}$ (Orlistat), and $0.04 \mu \mathrm{M}$ (Triacsin C), as shown in Figure 5B, D, F, and H, respectively. Importantly, the IC50s calculated for VPS34-IN1 and PIK-III by measuring infectious virus are in close agreement with IC50s calculated in Vero E6 cells using the resistance-based assay. The IC50s for Triacsin C and Orlistat were substantially lower than in 
271 the Vero cells. These data suggest that attenuation of the kinase activity of VPS34, synthesis of

272 fatty acids or production of long chain fatty acyl-CoA in human bronchial epithelial cells inhibits

273 replication of SARS-CoV-2.

VPS34 is a class III PI3 kinase. We therefore extended our study to determine if BYL719, an FDA approved inhibitor of class I PI3 kinase used to treat breast cancer, would also

276 inhibit SARS-CoV-2 replication in Calu-3 cells. Unlike the VPS34-specific inhibitors, little

277 inhibition was detected up to $16.6 \mu \mathrm{M}$, at which we observed a 1-log decrease in viral titers

278 (Supplemental Figure 2). This data suggests that not all PI3K classes play a significant role 279 during SARS-CoV-2 replication.

Inhibition of VPS34 kinase activity and fatty acid metabolism disperse SARS-CoV-2 replication centers autophagy membrane marker LC3 localizes ${ }^{8,9,23}$. We investigated if, similar to SARS-CoV-1 and MERS, SARS-CoV-2 nascent viral RNA and N co-localized with LC3. VeroE6 cells were infected with SARS-CoV-2 at a MOI of 3 and at 24 h.p.i., were treated with $1 \mu \mathrm{M}$ of actinomycin D to arrest host-cell transcription. Cells where then chased for 4 hours with 5-ethynyl uridine

287 (EU). Viral nascent RNA labeled during the EU chase was then detected with click chemistry, 288 indirect immunofluorescence performed using primary antibodies against $\mathrm{N}$ and LC3, and the 289 endoplasmic reticulum (ER) was detected with DPX BlueWhite ER stain. We observed distinct

290 formation of ring-like structures positive for ER, N, LC3, and nascent viral RNA (Supplemental 291 Figure 3A). Co-localization analysis demonstrated that nascent viral RNA co-localized with $\mathrm{N}$ 292 or LC3 (Supplemental Figure 3B). This data demonstrates the presence of SARS-CoV-2 293 replication centers that form in association with LC3. 
Because each compound exhibited inhibitory effects when added after viral entry, we

295 next asked whether the compounds altered the establishment of viral replication centers. Calu-3

296 cells were seeded onto fibronectin coated glass cover slips and allowed to reach 95\% confluency.

297 Cells were pre-treated with approximately the IC90 of VPS34-IN1 $(5 \mu \mathrm{M})$, PIK-III $(5 \mu \mathrm{M})$,

298 Orlistat $(500 \mu \mathrm{M})$, or Triacsin $\mathrm{C}(50 \mu \mathrm{M})$ and infected with SARS-CoV-2 at a MOI of 3 . At 24

299 h.p.i. cells were fixed, permeabilized, and indirect immunofluorescence performed using primary

300 antibodies against SARS-CoV-2 nucleoprotein (N) and dsRNA. We observed that when

301 compared to the media only or DMSO controls, $\mathrm{N}$ became completely cytoplasmic and did not

302 form any large inclusion like formations in the presence of the compounds (Figure 6).

303 Additionally, even though dsRNA could be detected both distributed throughout the cytoplasm

304 and associated with $\mathrm{N}$ in large inclusion like formations in the media only and DMSO controls,

305 in the cells treated with inhibitors, dsRNA was only found distributed throughout the cytoplasm.

306 This data suggests that the compound disrupt replication center formation.

\section{DISCUSSION}

308 Here, we demonstrate that two VPS34 inhibitors, Orlistat, and Triacsin C each have clear effects on SARS-CoV-2 replication and the morphology of viral replication centers. Generation of

310 replication centers is a key feature of the replication of many viruses ${ }^{36-38}$. These can serve as sites

311 where required components concentrate within a relatively closed environment and hide viral

312 replication products from the host innate immune response $\mathrm{s}^{39}$. In order to generate these centers,

313 many viruses usurp host cellular pathways that are used to generate membranes or organelles ${ }^{38}$.

314 Betacoronaviruses have been shown to target the ERAD-EDEMosome-ER pathways to generate

315 double-membrane vesicles required for their replication ${ }^{8}$. The data presented here suggests roles

316 for VPS34, FASN, and long chain fatty acyl CoA in replication center formation and stability 
suggesting a role for these host factors in providing the membranes needed for SARS-CoV-2

318 replication organelles.

VPS34 is of interest as a therapeutic target for a variety of conditions, including aging, neurodegeneration and cancer ${ }^{40,41}$. The two VPS34 inhibitors tested were VPS34-IN1 and PIK-

321 III which have in vitro IC50s for VPS34 of $25 \mathrm{nM}$ and $18 \mathrm{nM}$, respectively ${ }^{26,42}$. These were the 322 most potent compounds versus SARS-CoV-2 tested in this study. Each displayed an IC50 of less

323 than $1 \mu \mathrm{M}$ in either Vero E6 cells or Calu-3 cells. Activity in the Vero E6 cells was measured

324 based on the capacity of the compounds to prevent viral cytopathic effects as measured by

325 resistance across the cell monolayer, whereas the Calu-3 cell assay measured inhibition of 326 production of infectious virus particles. The resistance-based assay provided a built-in measure 327 of cell viability and integrity of the cell monolayer, providing assurance that decreases in 328 resistance measurements initially post-infection were not reflective of cytopathic effects. We also 329 independently determined that the compounds tested were non-toxic in Calu3 cells, likewise 330 demonstrating that decreases in viral titer were not due to compound toxicity. Based on the Calu3313 data, the selectivity indices (SI) (CC50/IC50) for the compounds are $>90$ and $>416$ for VPS34332 IN1 and PIK-III, respectively.

VPS34 is a phosphoinositide kinase that functions in autophagy, endosomal trafficking 334 and other cellular functions ${ }^{43}$. VPS34 associates with VPS15 as well as with other proteins to 335 carry out its activities. One VPS34-containing complex, Complex I, includes VPS34, VPS15, 336 Beclin 1 and ATG14 and is critical for autophagosome formation. Complex II includes VPS34, 337 VPS15, Beclin 1 and UVRAG and functions in autophagosome-lysosome fusion and in 338 regulation of endosomes and multivesicular bodies ${ }^{43}$. While our inhibitor studies do not 339 differentiate between the various functions of VPS34 that might be involved in SARS-CoV-2 
340 replication,. Autophagy has been implicated as necessary for MHV replication, however,

341 subsequent studies in different cell types suggest autophagy is not essential for MHV growth ${ }^{7,11}$.

342 Further, recent studies suggest that coronaviruses interfere with autophagy and that activation of

343 autophagy can inhibit replication of SARS-CoV, MERS CoV, and SARS-CoV-2 ${ }^{44,45}$. Given that

344 inhibition of VPS34 results in the inhibition of autophagy ${ }^{26,42}$, it would be expected that

345 inhibition of VPS34 would eliminate these anti-CoV effects of autophagy and promote SARS-

346 CoV-2 replication. Therefore, the disruptions in SARS-CoV-2 replication due to VPS34

347 inhibition described here may, instead, reflect inhibition of non-autophagy related functions of

$348 \quad$ VPS34.

349

Separate from autophagy, VPS34 has several other roles including in endosomal trafficking 350 and retrograde endosome-to-Golgi transport ${ }^{43}$. For the positive-sense RNA virus TBSV, VPS34

351 was implicated in providing phosphatidylethanolamine-enriched membranes for formation of

352 TBSV replication centers ${ }^{13}$. Based on our observation that VPS34 inhibitors disrupt the structure

353 of SARS-CoV-2 replication centers, it is possible that VPS34 functions to facilitate membrane

354 availability for SARS-CoV-2 replication organelle formation. Disruption of endocytic trafficking

355 might also explain our observation that pre-treatment with VPS34 inhibitors alone had 356 significant effects on SARS-CoV-2 replication.

357 Orlistat (tetrahydrolipstatin) is an FDA-approved weight loss drug that is taken orally and

358 inhibits gastric and pancreatic lipases in the digestive tract, reducing uptake of lipids ${ }^{21}$. Orlistat

359 also inhibits fatty acid synthase $(\mathrm{FASN})^{46}$. Orlistat and other FASN inhibitors have previously

360 been examined for their anti-cancer and antiviral activities. Although the clinically approved oral

361 administration of Orlistat does not result in its significant systemic distribution, pre-clinical

362 studies in mice have demonstrated that systemic administration of Orlistat is well tolerated ${ }^{47}$. 
363 Orlistat has been demonstrated to have activity against several viruses, including varicella-zoster

364 virus (VZV), coxsackievirus B3 virus (CVB3), dengue virus (DENV), and other flaviviruses.

365 DENV uses it nonstructural protein 3 to recruit FASN to viral replication sites and enhances

366 synthesis of fatty $\operatorname{acids}^{48}$. As in our study, flaviviruses were sensitive to relatively high

367 concentrations of Orlistat and antiviral effects could be demonstrated when Orlistat was added to

368 cells post-infection ${ }^{17}$. Virus inhibition has typically been demonstrated at relatively high

369 concentrations of Orlistat, such as $100 \mu \mathrm{M}$ or higher for CVB3, and between $10 \mu \mathrm{M}$ and $84 \mu \mathrm{M}$ for

370 DENV3, depending on the timepoint post-infection DENV3 replication was measured ${ }^{17,} 19,49$.

371 For DENV3, the effect of Orlistat appeared to be after the early stages of infection ${ }^{18}$. This may

372 reflect the need for DENV to recruit FASN to sites of virus replication and to upregulate fatty

373 acid synthesis ${ }^{48,50}$. It will be of interest to determine whether SARS-CoV-2 similarly depends on

374 an upregulation of fatty acid synthesis.

375 Triacsin $\mathrm{C}$ inhibits long chain fatty acid acyl-CoA synthetase. Interestingly, the long chain

376 fatty acid acyl-CoA synthetase ACSL3 was identified as an interactor of SARS-CoV-2 non-

377 structural protein 7 , suggesting a role for this enzyme in virus replication ${ }^{51}$. Triacsin $\mathrm{C}$ also has

378 demonstrated antiviral activity for $\mathrm{HCV}$ and rotavirus ${ }^{14-16}$. For both HCV and rotavirus, the

379 antiviral effects of Triacsin $\mathrm{C}$ have been linked to reliance of these viruses on lipid droplets for

380 their replication ${ }^{14-16}$. Lipid droplets are organelles that store neutral lipids of which triglycerides

381 are a major component ${ }^{52}$. By inhibiting long chain fatty acyl CoA, Triacsin C blocks lipid droplet

382 formation. That antiviral activity against $\mathrm{HCV}$ and rotavirus is connected to lipid droplet

383 formation is supported by the fact that these viruses are sensitive to inhibition by the DGAT

384 inhibitors, T863 and PF06424439. In contrast, the compounds did not exhibit any activity against

385 SARS-CoV-2 in Vero E6 cells whereas Triacsin C did. This suggests an alternate role for long 
chain fatty acyl CoA or its downstream metabolites other than triacylglycerol and lipid droplets.

387 It is notable that the IC50 for Triacsin C was substantially lower in the Calu-3 cell assay as

388 compared to the Vero cell assay. A lesser decrease in IC50 was also noted for Orlistat in the

389 Calu-3 cells versus the Vero E6 cells. These observations may reflect different degrees of

390 dependence of the virus on fatty acid metabolism in different cell types. From the perspective of

391 antiviral development, it is encouraging that the human airway-derived cells are the more

392 sensitive system given that SARS-CoV-2 targets the respiratory tract. Triacsin $\mathrm{C}$ has been

393 administered to mice daily for up to two months without overt signs of significant toxicity and

394 resulted in a decrease in atherosclerosis ${ }^{53}$. However, the pharmacokinetics and cell penetrance of

395 Triacsin $\mathrm{C}$ are viewed as significant impediments to its clinical use ${ }^{54}$. Despite this, Triacsin C

396 analogs have been developed ${ }^{15}$, and long chain fatty acyl CoA synthetases are of interest as

397 potential therapeutics for cancer as well as for viruses ${ }^{54}$.

398 Cumulatively, these data support lipid metabolism as a potential therapeutic target for SARS-

399 CoV-2 infection. The specific mechanisms by which VPS34 promotes SARS-CoV-2 replication

400 and the precise manner in which the VSP34 inhibitors impair replication warrant further

401 investigation. Additionally, the specific enzymes and products of fatty acid metabolism

402 necessary for efficient SARS-CoV-2 growth in human airway epithelial cells should be further

403 explored to more precisely identify relevant targets for therapeutic targeting. Further, it will be of

404 interest to understand the relative efficacies of inhibitors of fatty acid metabolism in different cell

405 types. 
407 Acknowledgments. This work was supported by NIH grants R01AI125453 and P01AI120943

408 (Amarasinghe) to CFB. We would like to thank the Georgia State University High Containment

409 team Natasha Griffith, Martin Wildes, and Robert "Mike” Walsh for their continuous support.

411 Competing Interests. Authors A.M.N. and S.A.C. are employees of Axion BioSystems who 412 provided the Axion Maestro $\mathrm{Z}$ instrument used in these studies. 


\section{References}

415

1. Lundstrom, K. Coronavirus Pandemic-Therapy and Vaccines. Biomedicines 8 (2020).

2. Wang, L., Wang, Y., Ye, D. \& Liu, Q. Review of the 2019 novel coronavirus (SARSCoV-2) based on current evidence. Int J Antimicrob Agents 55, 105948 (2020).

3. García-Serradilla, M., Risco, C. \& Pacheco, B. Drug repurposing for new, efficient, broad spectrum antivirals. Virus Res 264, 22-31 (2019).

4. Pizzorno, A., Padey, B., Terrier, O. \& Rosa-Calatrava, M. Drug Repurposing Approaches for the Treatment of Influenza Viral Infection: Reviving Old Drugs to Fight Against a Long-Lived Enemy. Front Immunol 10, 531 (2019).

5. Saini, K.S. et al. Repurposing anticancer drugs for COVID-19-induced inflammation, immune dysfunction, and coagulopathy. Br J Cancer (2020).

6. Li, G. \& De Clercq, E. Therapeutic options for the 2019 novel coronavirus (2019-nCoV). Nat Rev Drug Discov 19, 149-150 (2020).

7. Prentice, E., Jerome, W.G., Yoshimori, T., Mizushima, N. \& Denison, M.R. Coronavirus replication complex formation utilizes components of cellular autophagy. J Biol Chem 279, 10136-10141 (2004).

8. Reggiori, F. et al. Coronaviruses Hijack the LC3-I-positive EDEMosomes, ER-derived vesicles exporting short-lived ERAD regulators, for replication. Cell Host Microbe 7, 500-508 (2010).

9. Reggiori, F., de Haan, C.A. \& Molinari, M. Unconventional use of LC3 by coronaviruses through the alleged subversion of the ERAD tuning pathway. Viruses 3, 1610-1623 (2011).

10. Snijder, E.J. et al. A unifying structural and functional model of the coronavirus replication organelle: Tracking down RNA synthesis. PLoS Biol 18, e3000715 (2020).

11. Zhao, Z. et al. Coronavirus replication does not require the autophagy gene ATG5. Autophagy 3, 581-585 (2007).

12. Su, W.C. et al. Rab5 and class III phosphoinositide 3-kinase Vps34 are involved in hepatitis C virus NS4B-induced autophagy. J Virol 85, 10561-10571 (2011).

13. Feng, Z., Xu, K., Kovalev, N. \& Nagy, P.D. Recruitment of Vps34 PI3K and enrichment of PI3P phosphoinositide in the viral replication compartment is crucial for replication of a positive-strand RNA virus. PLoS Pathog 15, e1007530 (2019).

14. Liefhebber, J.M., Hague, C.V., Zhang, Q., Wakelam, M.J. \& McLauchlan, J. Modulation of triglyceride and cholesterol ester synthesis impairs assembly of infectious hepatitis $\mathrm{C}$ virus. J Biol Chem 289, 21276-21288 (2014).

15. Kim, Y. et al. Novel triacsin $\mathrm{C}$ analogs as potential antivirals against rotavirus infections. Eur J Med Chem 50, 311-318 (2012).

16. Cheung, W. et al. Rotaviruses associate with cellular lipid droplet components to replicate in viroplasms, and compounds disrupting or blocking lipid droplets inhibit viroplasm formation and viral replication. J Virol 84, 6782-6798 (2010).

17. Hitakarun, A. et al. Evaluation of the antiviral activity of orlistat (tetrahydrolipstatin) against dengue virus, Japanese encephalitis virus, Zika virus and chikungunya virus. Sci Rep 10, 1499 (2020).

18. Tongluan, N. et al. Involvement of fatty acid synthase in dengue virus infection. Virol $J$ 14, 28 (2017). 
19. Ammer, E. et al. The anti-obesity drug orlistat reveals anti-viral activity. Med Microbiol Immunol 204, 635-645 (2015).

20. Esser, K. et al. Lipase inhibitor orlistat prevents hepatitis B virus infection by targeting an early step in the virus life cycle. Antiviral Res 151, 4-7 (2018).

21. Heck, A.M., Yanovski, J.A. \& Calis, K.A. Orlistat, a new lipase inhibitor for the management of obesity. Pharmacotherapy 20, 270-279 (2000).

22. Benson, K., Cramer, S. \& Galla, H.J. Impedance-based cell monitoring: barrier properties and beyond. Fluids Barriers CNS 10, 5 (2013).

23. Prentice, E., McAuliffe, J., Lu, X., Subbarao, K. \& Denison, M.R. Identification and characterization of severe acute respiratory syndrome coronavirus replicase proteins. $J$ Virol 78, 9977-9986 (2004).

24. Gordon, C.J. et al. Remdesivir is a direct-acting antiviral that inhibits RNA-dependent RNA polymerase from severe acute respiratory syndrome coronavirus 2 with high potency. J Biol Chem 295, 6785-6797 (2020).

25. Wu, J., Wu, B. \& Lai, T. Compassionate Use of Remdesivir in Covid-19. N Engl J Med 382 (2020).

26. Bago, R. et al. Characterization of VPS34-IN1, a selective inhibitor of Vps34, reveals that the phosphatidylinositol 3-phosphate-binding SGK3 protein kinase is a downstream target of class III phosphoinositide 3-kinase. Biochem J 463, 413-427 (2014).

27. Wakil, S.J. \& Abu-Elheiga, L.A. Fatty acid metabolism: target for metabolic syndrome. $J$ Lipid Res 50 Suppl, S138-143 (2009).

28. Schütter, M., Giavalisco, P., Brodesser, S. \& Graef, M. Local Fatty Acid Channeling into Phospholipid Synthesis Drives Phagophore Expansion during Autophagy. Cell 180, 135149.e114 (2020).

29. Bakhache, W. et al. Fatty acid synthase and stearoyl-CoA desaturase-1 are conserved druggable cofactors of Old World Alphavirus genome replication. Antiviral Res 172, 104642 (2019).

30. Nasheri, N. et al. Modulation of fatty acid synthase enzyme activity and expression during hepatitis $\mathrm{C}$ virus replication. Chem Biol 20, 570-582 (2013).

31. Herker, E. et al. Efficient hepatitis $\mathrm{C}$ virus particle formation requires diacylglycerol acyltransferase-1. Nat Med 16, 1295-1298 (2010).

32. Cao, J. et al. Targeting Acyl-CoA:diacylglycerol acyltransferase 1 (DGAT1) with small molecule inhibitors for the treatment of metabolic diseases. J Biol Chem 286, 4183841851 (2011).

33. Futatsugi, K. et al. Discovery and Optimization of Imidazopyridine-Based Inhibitors of Diacylglycerol Acyltransferase 2 (DGAT2). J Med Chem 58, 7173-7185 (2015).

34. Sims, A.C. et al. Severe acute respiratory syndrome coronavirus infection of human ciliated airway epithelia: role of ciliated cells in viral spread in the conducting airways of the lungs. J Virol 79, 15511-15524 (2005).

35. Sims, A.C., Burkett, S.E., Yount, B. \& Pickles, R.J. SARS-CoV replication and pathogenesis in an in vitro model of the human conducting airway epithelium. Virus Res 133, 33-44 (2008).

36. Nagy, P.D., Strating, J.R. \& van Kuppeveld, F.J. Building Viral Replication Organelles: Close Encounters of the Membrane Types. PLoS Pathog 12, e1005912 (2016).

37. Sasvari, Z. \& Nagy, P.D. Making of viral replication organelles by remodeling interior membranes. Viruses 2, 2436-2442 (2010). 
504

505

506

507

508

509

510

511

512

513

514

515

516

517

518

519

520

521

522

523

524

525

526

527

528

529

530

531

532

533

534

535

536

537

538

539

540

541

542

38. den Boon, J.A. \& Ahlquist, P. Organelle-like membrane compartmentalization of positive-strand RNA virus replication factories. Annu Rev Microbiol 64, 241-256 (2010).

39. Santiago, F.W. et al. Hijacking of RIG-I signaling proteins into virus-induced cytoplasmic structures correlates with the inhibition of type I interferon responses. $J$ Virol 88, 4572-4585 (2014).

40. Morris, D.H., Yip, C.K., Shi, Y., Chait, B.T. \& Wang, Q.J. Beclin 1-Vps34 Complex Architecture: Understanding the Nuts and Bolts of Therapeutic Targets. Front Biol (Beijing) 10, 398-426 (2015).

41. Chude, C.I. \& Amaravadi, R.K. Targeting Autophagy in Cancer: Update on Clinical Trials and Novel Inhibitors. Int J Mol Sci 18 (2017).

42. Dowdle, W.E. et al. Selective VPS34 inhibitor blocks autophagy and uncovers a role for NCOA4 in ferritin degradation and iron homeostasis in vivo. Nat Cell Biol 16, 1069-1079 (2014).

43. Backer, J.M. The intricate regulation and complex functions of the Class III phosphoinositide 3-kinase Vps34. Biochem J 473, 2251-2271 (2016).

44. Guo, L. et al. Autophagy Negatively Regulates Transmissible Gastroenteritis Virus Replication. Sci Rep 6, 23864 (2016).

45. Gassen, N.C. et al. SKP2 attenuates autophagy through Beclin1-ubiquitination and its inhibition reduces MERS-Coronavirus infection. Nat Commun 10, 5770 (2019).

46. Wakil, S.J. Fatty acid synthase, a proficient multifunctional enzyme. Biochemistry 28, 4523-4530 (1989).

47. Schcolnik-Cabrera, A. et al. Orlistat as a FASN inhibitor and multitargeted agent for cancer therapy. Expert Opin Investig Drugs 27, 475-489 (2018).

48. Heaton, N.S. et al. Dengue virus nonstructural protein 3 redistributes fatty acid synthase to sites of viral replication and increases cellular fatty acid synthesis. Proc Natl Acad Sci $U S A$ 107, 17345-17350 (2010).

49. Wilsky, S. et al. Inhibition of fatty acid synthase by amentoflavone reduces coxsackievirus B3 replication. Arch Virol 157, 259-269 (2012).

50. Tang, W.C., Lin, R.J., Liao, C.L. \& Lin, Y.L. Rab18 facilitates dengue virus infection by targeting fatty acid synthase to sites of viral replication. J Virol 88, 6793-6804 (2014).

51. Gordon, D.E. et al. A SARS-CoV-2 protein interaction map reveals targets for drug repurposing. Nature 583, 459-468 (2020).

52. Olzmann, J.A. \& Carvalho, P. Dynamics and functions of lipid droplets. Nat Rev Mol Cell Biol 20, 137-155 (2019).

53. Matsuda, D. et al. Anti-atherosclerotic activity of triacsin C, an acyl-CoA synthetase inhibitor. J Antibiot (Tokyo) 61, 318-321 (2008).

54. Rossi Sebastiano, M. \& Konstantinidou, G. Targeting Long Chain Acyl-CoA Synthetases for Cancer Therapy. Int J Mol Sci 20 (2019). 
546 CoV-2 induced CPE and anti-SARS-CoV-2 activity. VeroE6 cells were seeded into a

547 CytoView-Z 96-well plate and cells were allowed to stabilize overnight, as measured by

548 electrical resistance. A) SARS-CoV-2 was titrated in 10-fold dilutions ranging from 10-0.0001

549 MOI. Resistance was measured every minute over the course of 72 hours. Solid lines indicate the

550 mean, dotted lines indicate the standard error of three replicates. B) Median time to death

551 calculations based on raw resistance data for each MOI. C) Remdesivir was titrated in 6-fold

552 dilutions ranging from 50-0.006 $\mu \mathrm{M}$. After infection at an MOI of 0.01 , resistance was

553 monitored for 48 h.p.i. and D) percent inhibition was determined at the 48 hour timepoint.

555 Figure 2. VPS34 inhibitors exhibit anti-SARS-CoV-2 activity. VeroE6 cells were seeded into

556 a CytoView-Z 96-well plate, and cells were allowed to stabilize overnight. Cells were pre-treated

557 with serial half-log dilutions of A) VPS34-IN1 or C) PIK-III and infected with SARS-CoV-2 at

558 an $\mathrm{MOI}=0.01$. Resistance (A and $\mathbf{C}$ ) was measured every minute over the course of 48 hours and

559 percent inhibition (B and D) was determined at the 48-hour timepoint. Solid lines indicate mean, 560 dotted lines indicate the standard error of two replicates.

562 Figure 3. Screening of fatty acid inhibitors for potential anti-SARS-CoV-2 activity. VeroE6

563 cells were seeded into a CytoView-Z 96-well plate and allowed to stabilize overnight. Cells were

564 pre-treated with serial half-log dilutions of A) Orlistat or B) Triacsin C and infected with SARS-

$565 \mathrm{CoV}-2$ at an $\mathrm{MOI}=0.01$. Resistance (A and C) was measured every minute over the course of 48 
566 hours and percent inhibition (B and D) was determined at the 48-hour timepoint. Solid lines

567 indicate the mean and dotted lines indicate the standard error of two replicates.

Figure 4. Single treatment of VPS34 inhibitors have potent anti-viral activity against

570 SARS-CoV-2. VeroE6 cells were seeded into a CytoView-Z 96-well plate, and allowed to

571 stabilize overnight. A) Timeline for the time-of-addition experiment. B) VeroE6 cells were pre-

572 treated for one hour and compound was removed (-1), pre-treated for one hour with compound

573 maintained throughout infection $(+1)$, or treated at $2(+2)$ or $4(+4)$ hours post-infection with an

574 MOI of 0.01 . Resistance was measured every minute over the course of 48 hours and percent

575 inhibition was determined at the 48-hour timepoint. Data is representative of the mean and

576 standard error of three technical replicates.

578 Figure 5. Attenuation of VPS34 kinase activity and fatty acid metabolism inhibit SARS-

579 CoV-2 replication in human airway epithelial cell line. Calu-3 cells were plated onto a 96-

580 well plate and allowed to reach $95 \%$ confluency. Cells were then pre-treated with a range of

581 concentrations of A-B) VPS34-IN1, C-D) PIK-III, E-F) Orlistat, G-H), Triacsin C, DMSO, or

582 mock-treated with media alone for 1 hour then infected with SARS-CoV-2 at an MOI of 0.01.

583 Supernatants were collected at 48 h.p.i. and virus was quantified by plaque assay on VeroE6

584 cells. The data is reported as plaque forming units per milliliter (pfu/ml) (left panels). Cell

585 viability over 48 hours was determined in parallel. Percent inhibition, IC50, and IC90 were

586 calculated from the plaque assay data and plotted with the cell viability data (right panels). The 
587 dotted line labeled DMSO indicates the level of virus growth in the DMSO control. The dotted

588 line labeled LOD indicates the limit of detection of the plaque assay.

592 Orlistat $(500 \mathrm{uM})$, or Triacsin $\mathrm{C}(50 \mathrm{uM})$ for 1 hour and infected with SARS-CoV-2 at MOI of

593 0.01. Cells were fixed at 24 h.p.i. and immunofluorescence was performed using primary

594 antibodies against SARS-CoV-2 $\mathrm{N}$ or dsRNA, and AlexaFluor488 or AlexaFluor647 conjugated

595 secondary antibodies, respectively. Nuclei were stained with Hoeschst 33342. Representative

596 images are shown.

599 VeroE6 cells were seeded into a CytoView-Z 96-well plate and allowed to stabilize overnight.

600 Cells were pre-treated with serial half-log dilutions of A) TC863 or B) PF06424439 and infected 601 with SARS-CoV-2 at an MOI=0.01. Resistance was measured every minute over the course of 60248 hours and percent inhibition relative to the DMSO control was determined at the 48-hour 603 timepoint.

605 Supplemental Figure 2. Inhibition of alpha PI3K does not prevent SARS-CoV-2 replication. Calu-3 cells were plated onto a 96-microplate and allowed to reach $95 \%$ confluency.

607 Cells were then pre-treated with a range of concentrations of BYL719 and infected with SARS- 
609 (left panel). Cell toxicity was determined in parallel and percent inhibition extrapolated from 610 plaque assay data (right panel).

612 Supplemental Figure 3. SARS-CoV-2 N and nascent viral RNA co-localize with the

613 autophagy membrane marker LC3. VeroE6 cells were infected with SARS-CoV-2. At 24

614 h.p.i., cells were pre-treated with actinomycin D followed by a 5-ethynyl uridine (EU) chase for

6154 hours. A) Cells were fixed, EU labeled viral nascent RNA was detected with click chemistry, 616 and immunofluorescence performed using primary antibodies against SARS-CoV-2 N or LC3

617 and AlexaFluor488- or AlexaFluor647- conjugated secondary antibodies, respectively. Nuclei

618 were stained with Hoeschst 33342. Representative images are shown. B) Co-localization was 619 analyzed with Zen Blue. 
bioRxiv preprint doi: https://doi.org/10.1101/2020.07.18.210211; this ver IODposted July 20, 2020. The copyright holder for this preprint (which was not certified by peer review) is the author/funder, who has grant anjoRxiv a license to display the preprint in perpetuity. It is made available under aCC-BY-NC-N 4 . International license.

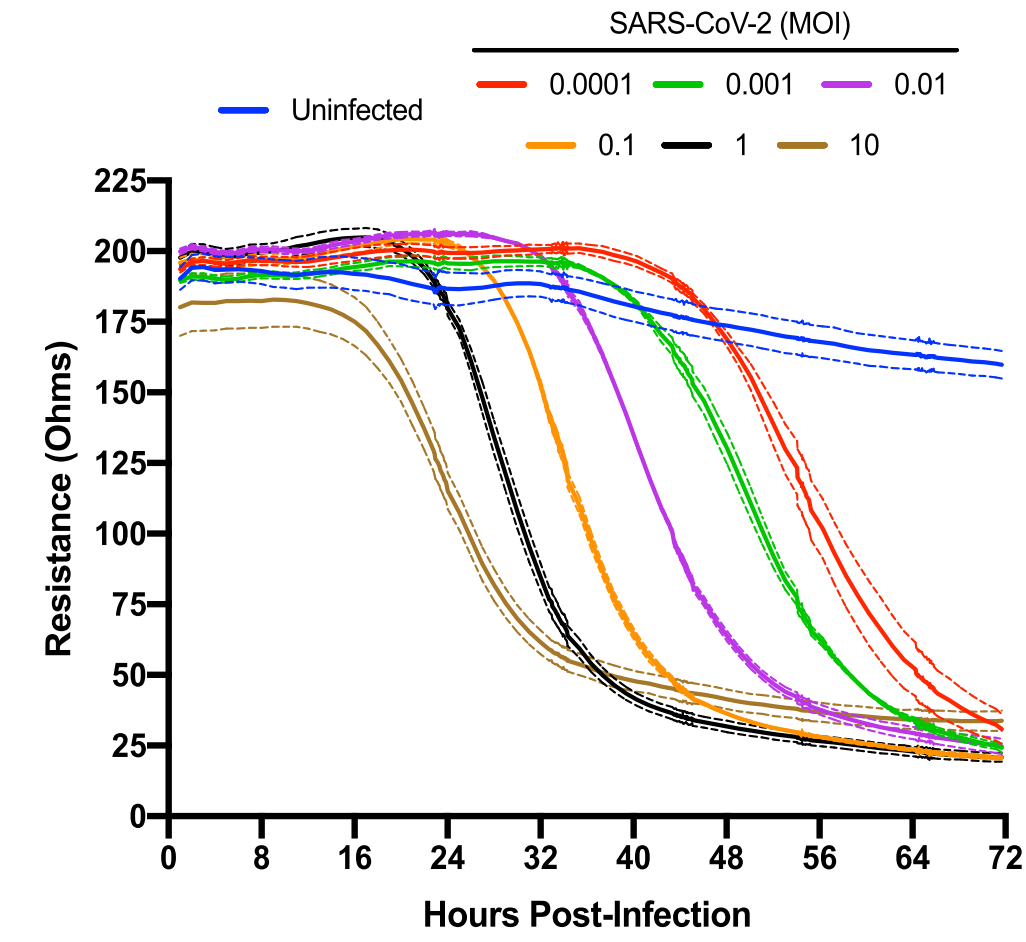

C.
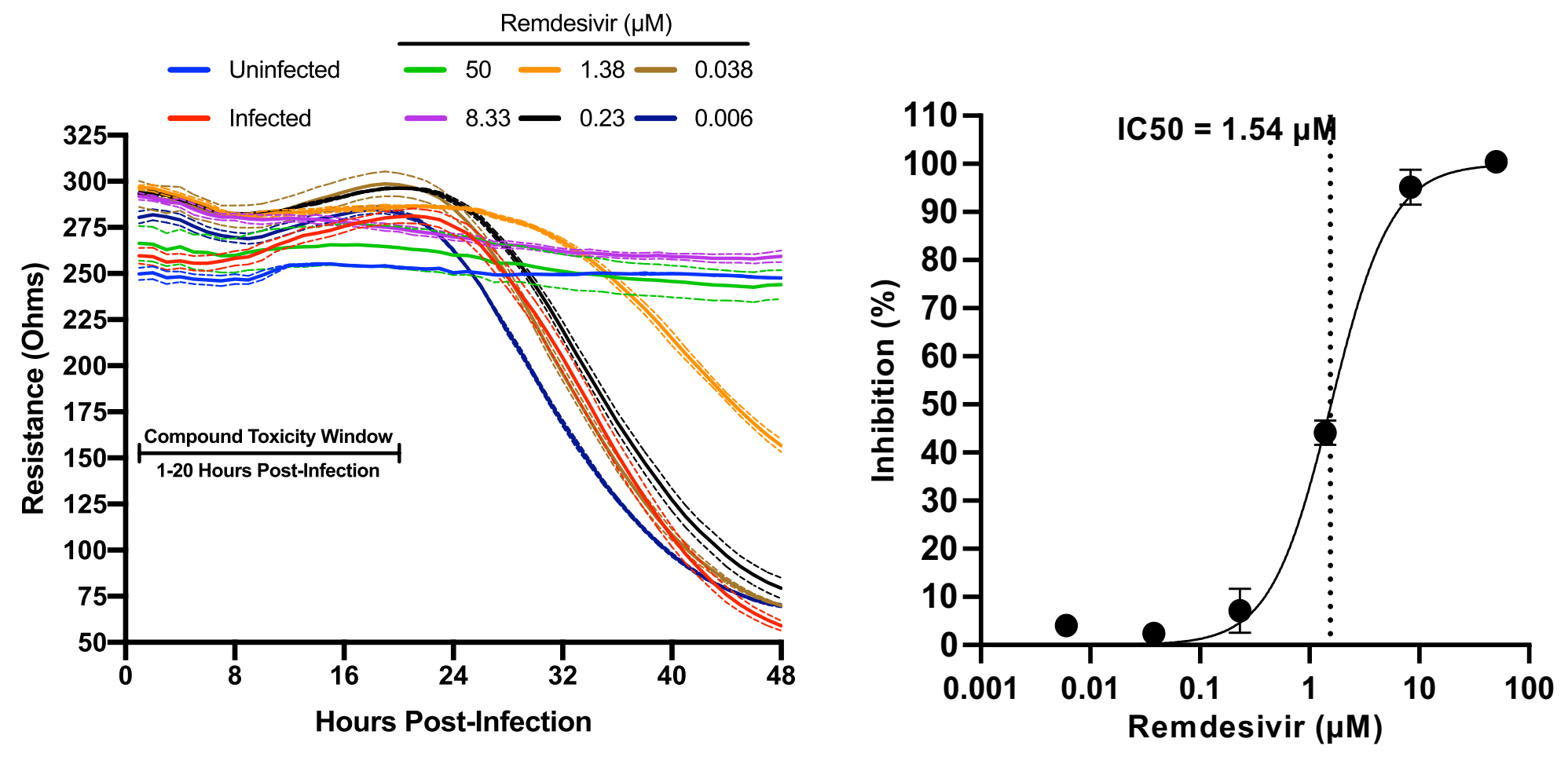


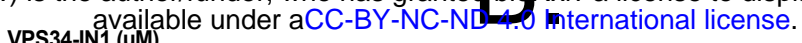
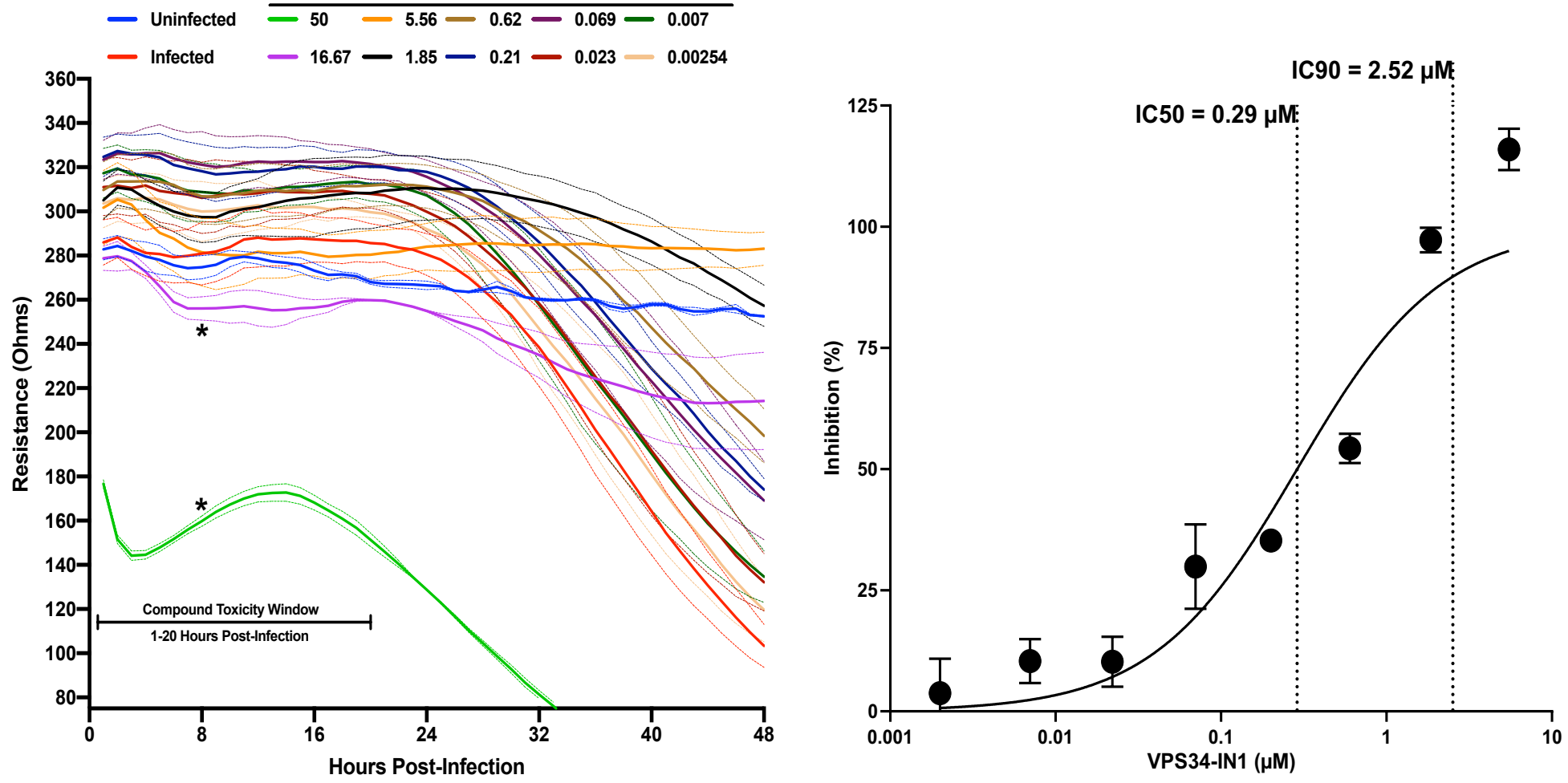

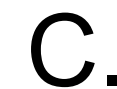
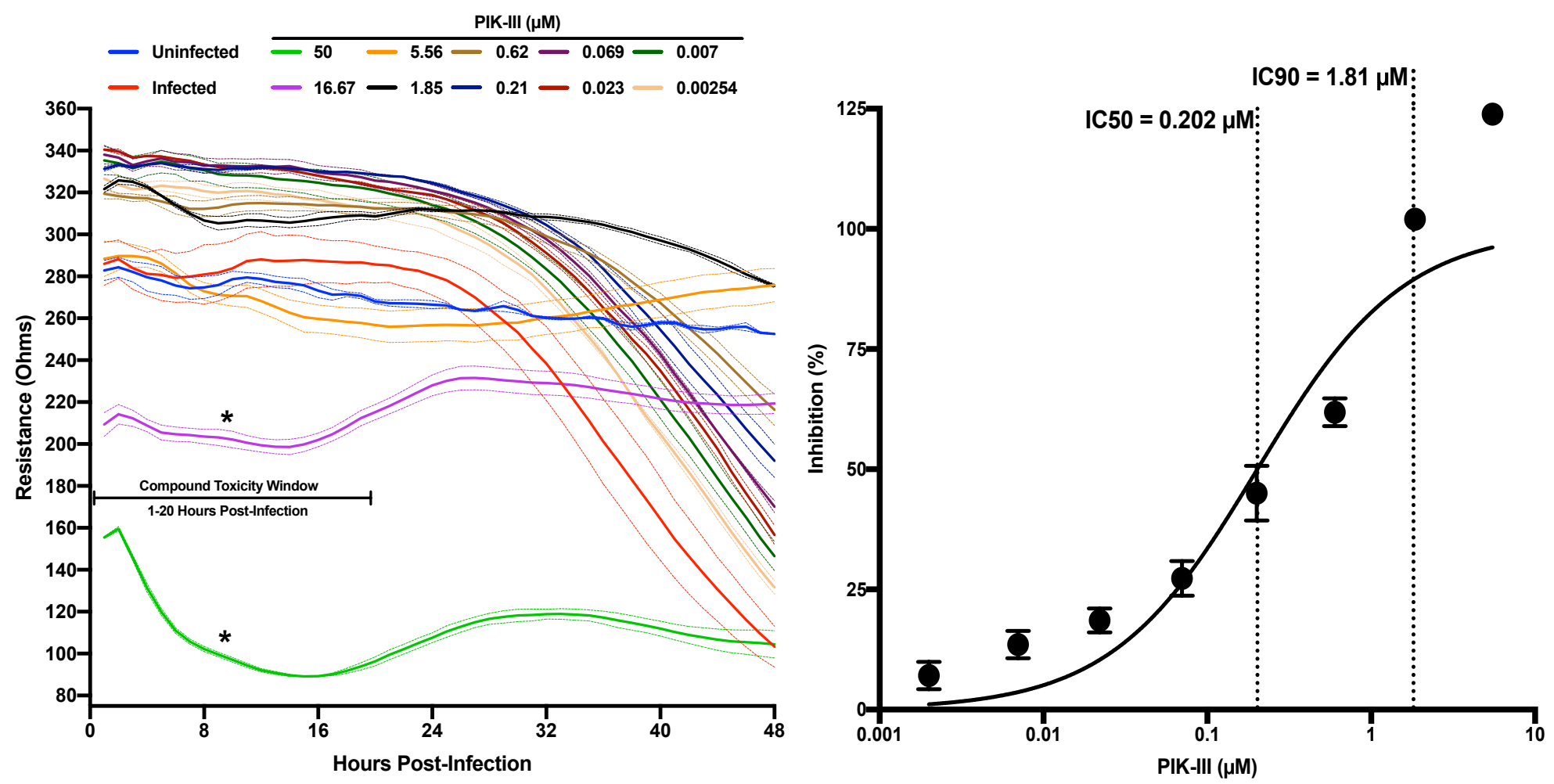
bioRxiv preprint doi: https://doi.org/10.1101/2020.07.18.210211; this versi $\mathbf{M}$ osted July 20,2020 . The copyright holder for this preprint (which was not certified by peer review) is the author/funder, who has grante Orlistat $(\mu \mathrm{M})$ available under aCC-BY-NC-NL 1.0 Rxiv a license to display

display the preprint in perpetuity. It is made $-55.56-6.17-0.69-0.08$
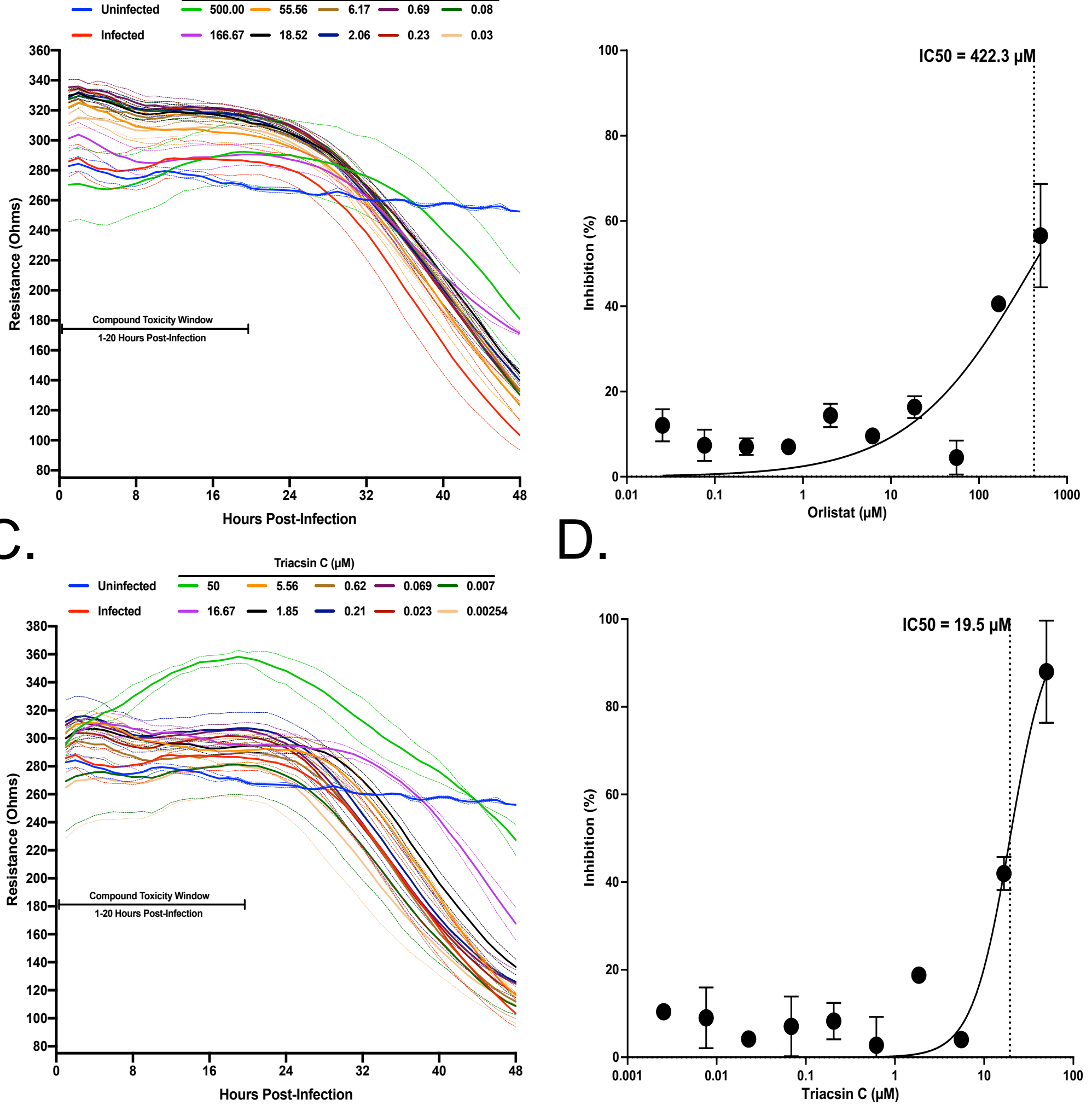
bioRxiv preprint doi: https://doi.org/10.1101/2020.07.18.210211; this version posted July 20, 2020. The copyright holder for this preprint (which was not certified by peer review) is the author/funder, who has granted bioRxiv a license to display the preprint in perpetuity. It is made

A. Virus Addition

Compound

Treatment available under aCC-BY-NC-ND 4.0 International license.

Additio
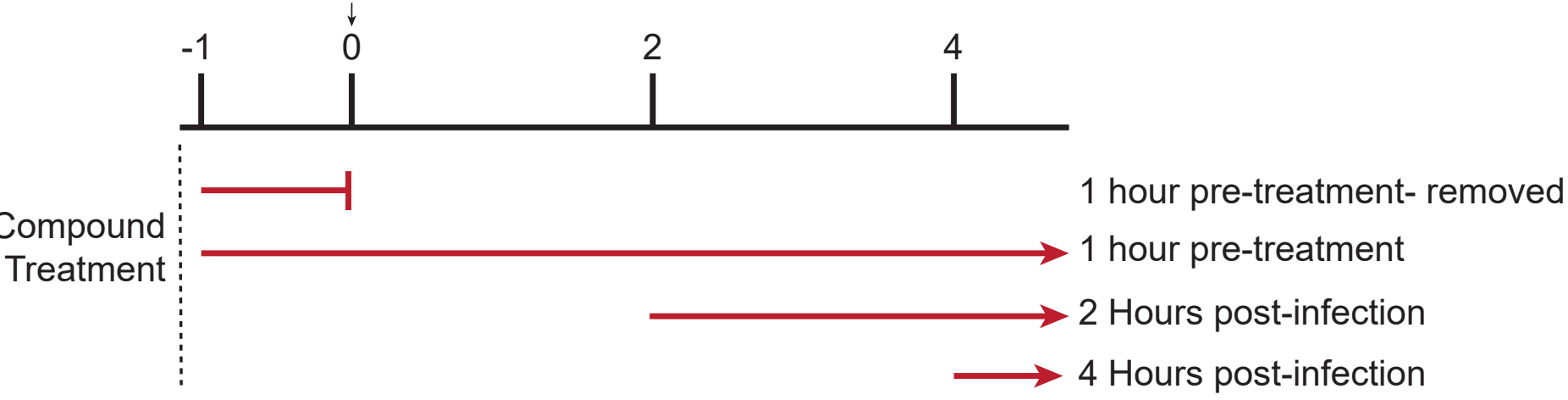

B.

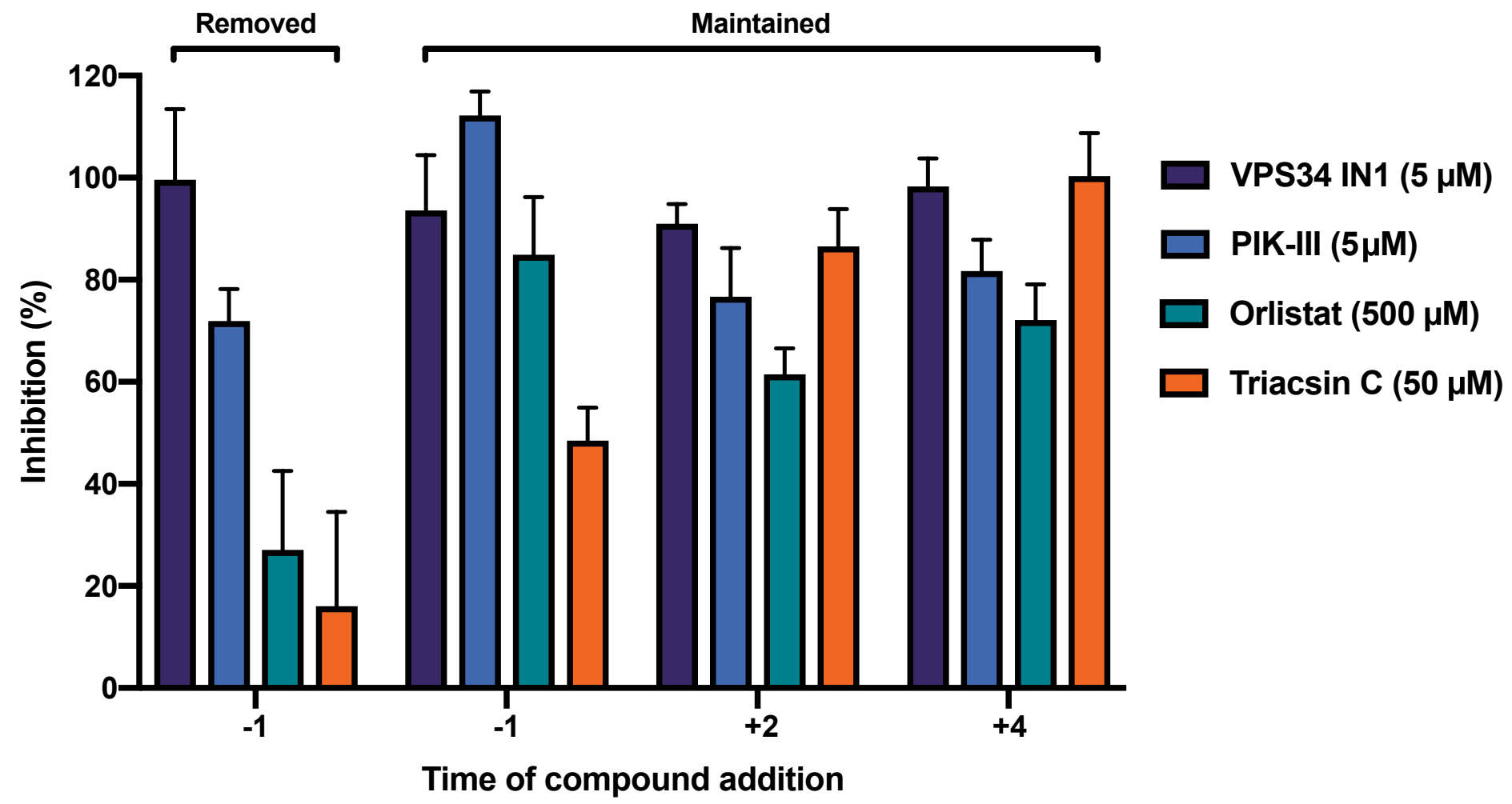


bioRxi106reprint doi: https://doi.org/10.1101/2020.07.18210211: this version posted July 20, 2020. The copyright holder for this preprint (which

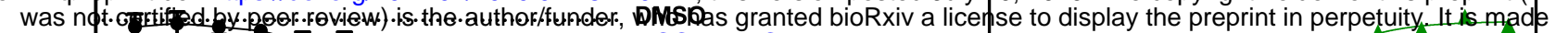
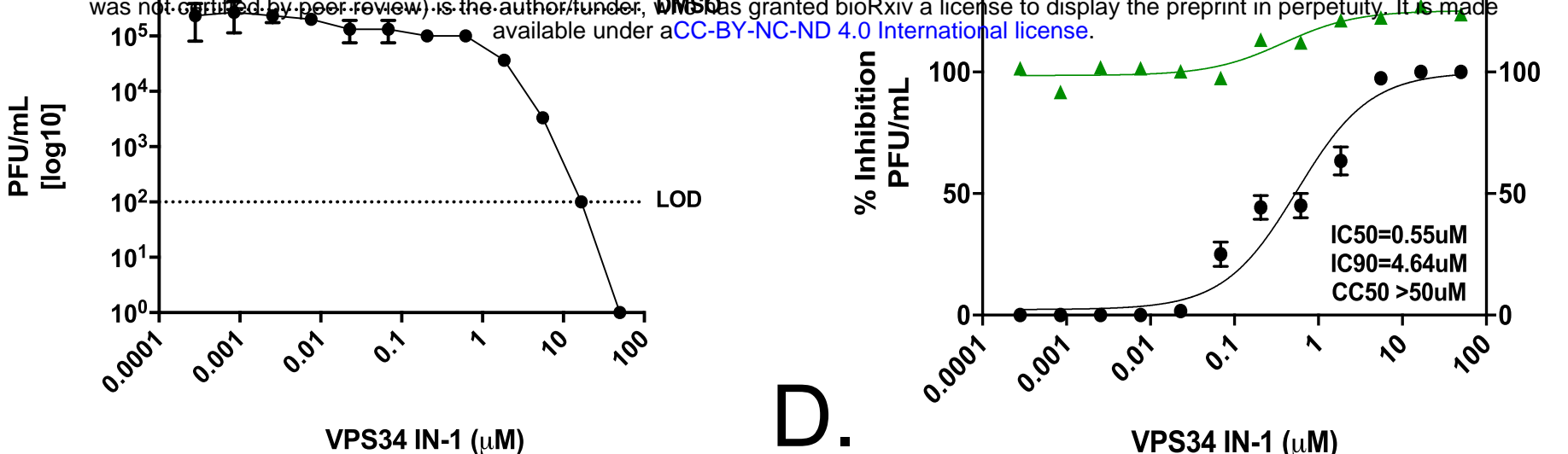

VPS34 IN-1 (uM)

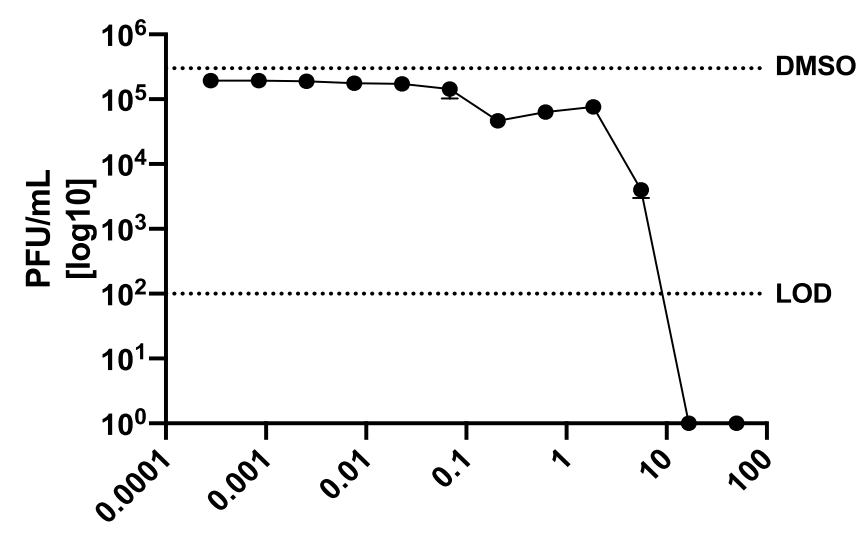

PIK-III $(\mu \mathrm{M})$

$E$

$G$.

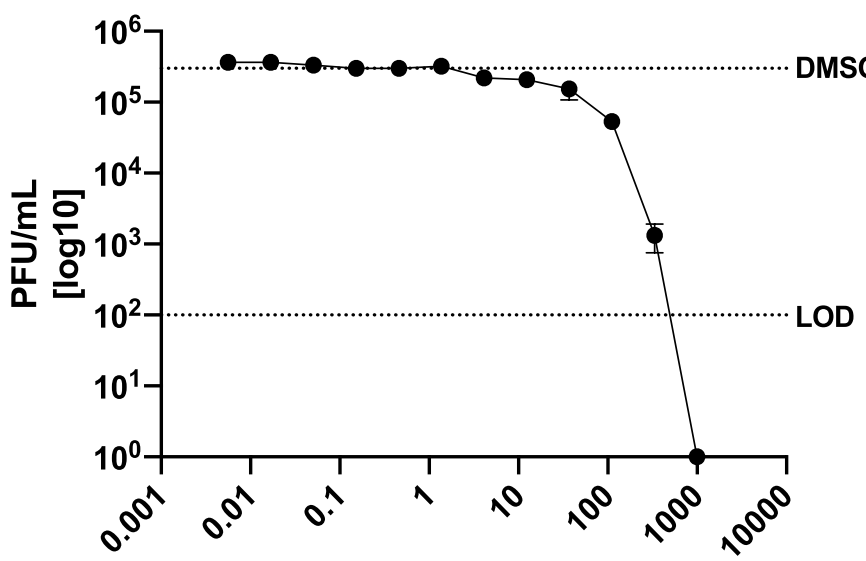

Orlistat $(\mu M)$

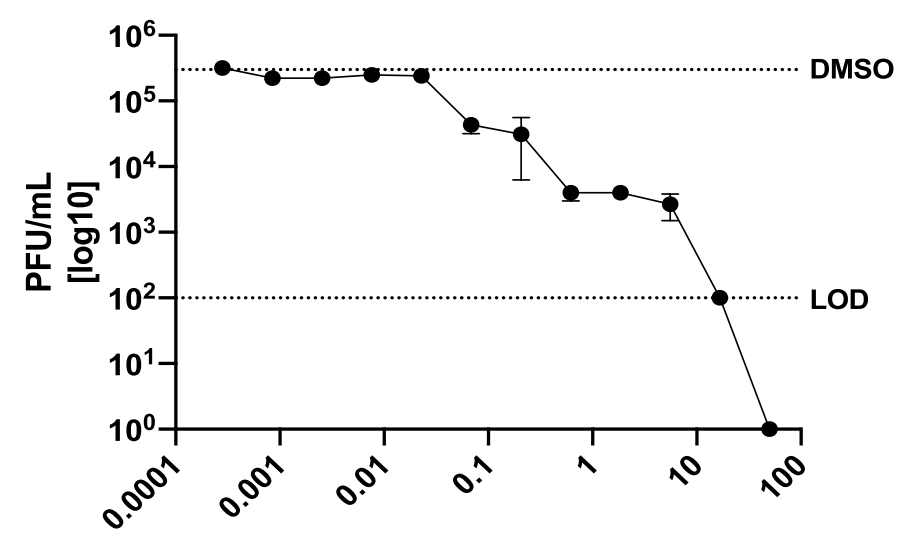

Triacsin C $(\mu \mathrm{M})$

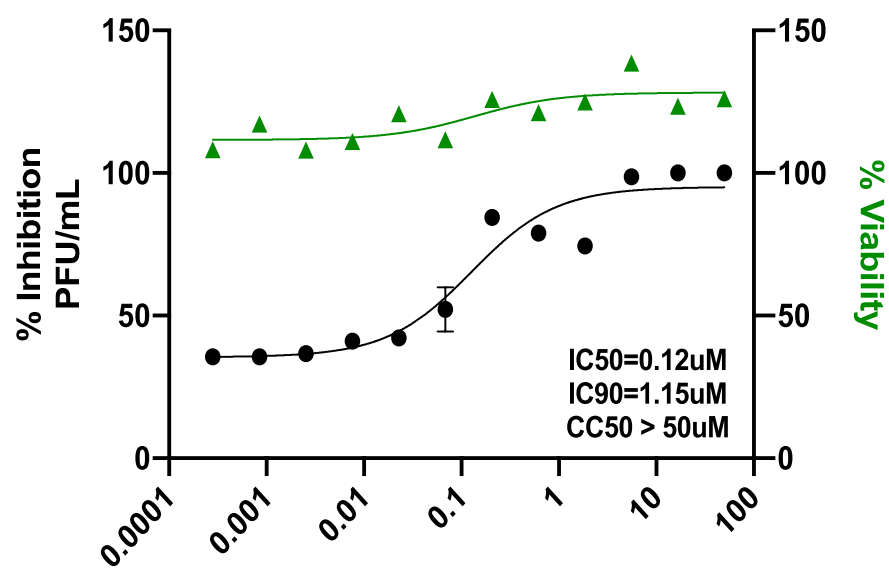

PIK-III ( $\mu \mathrm{M})$

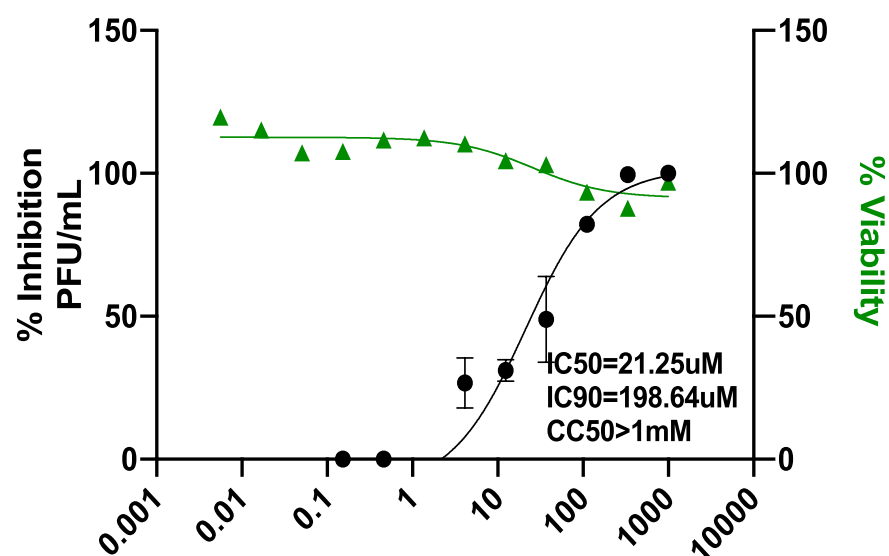

Orlistat $(\mu \mathrm{M})$

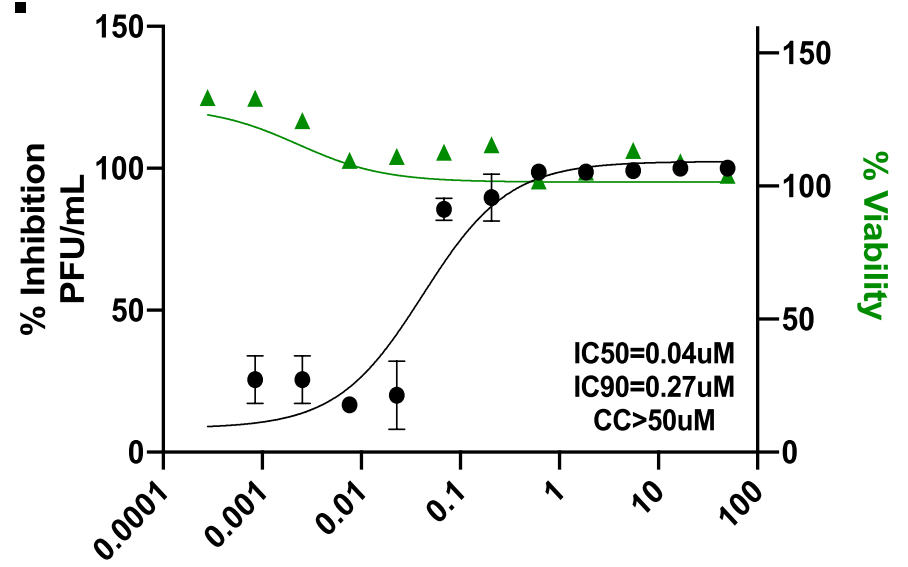

Triacsin $\mathrm{C}(\mu \mathrm{M})$ 

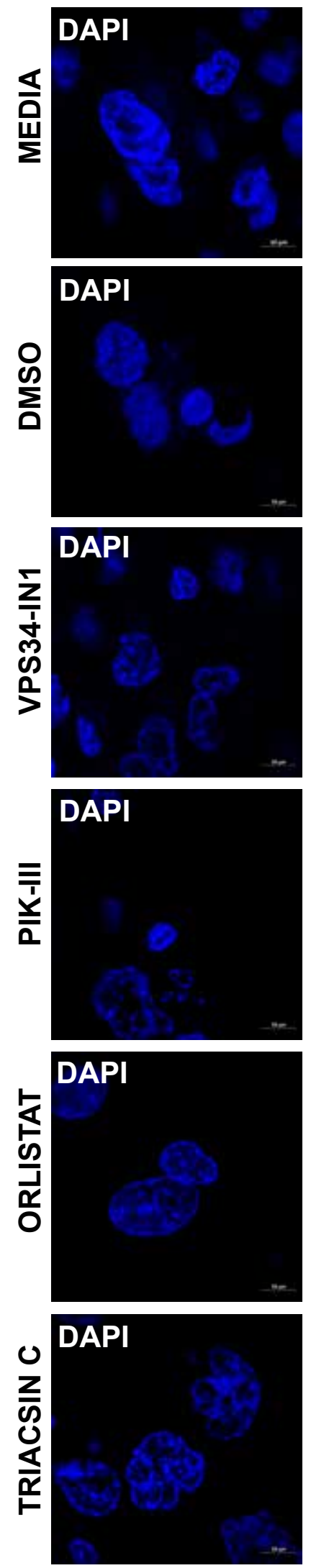
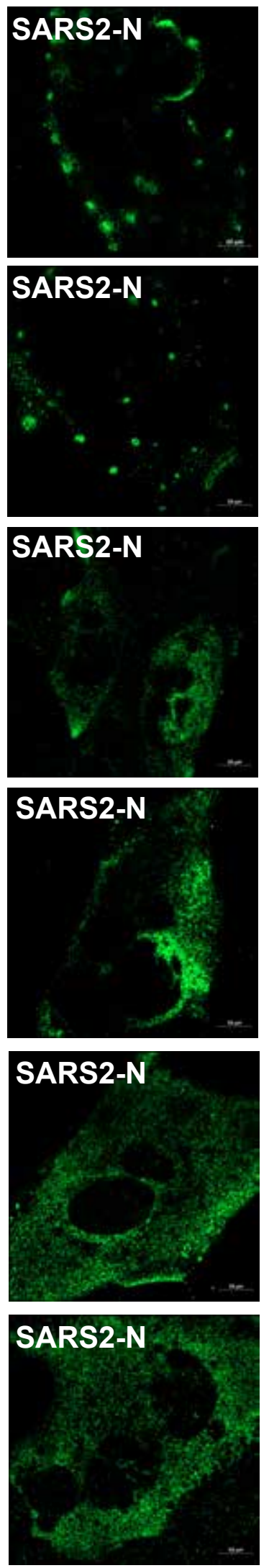
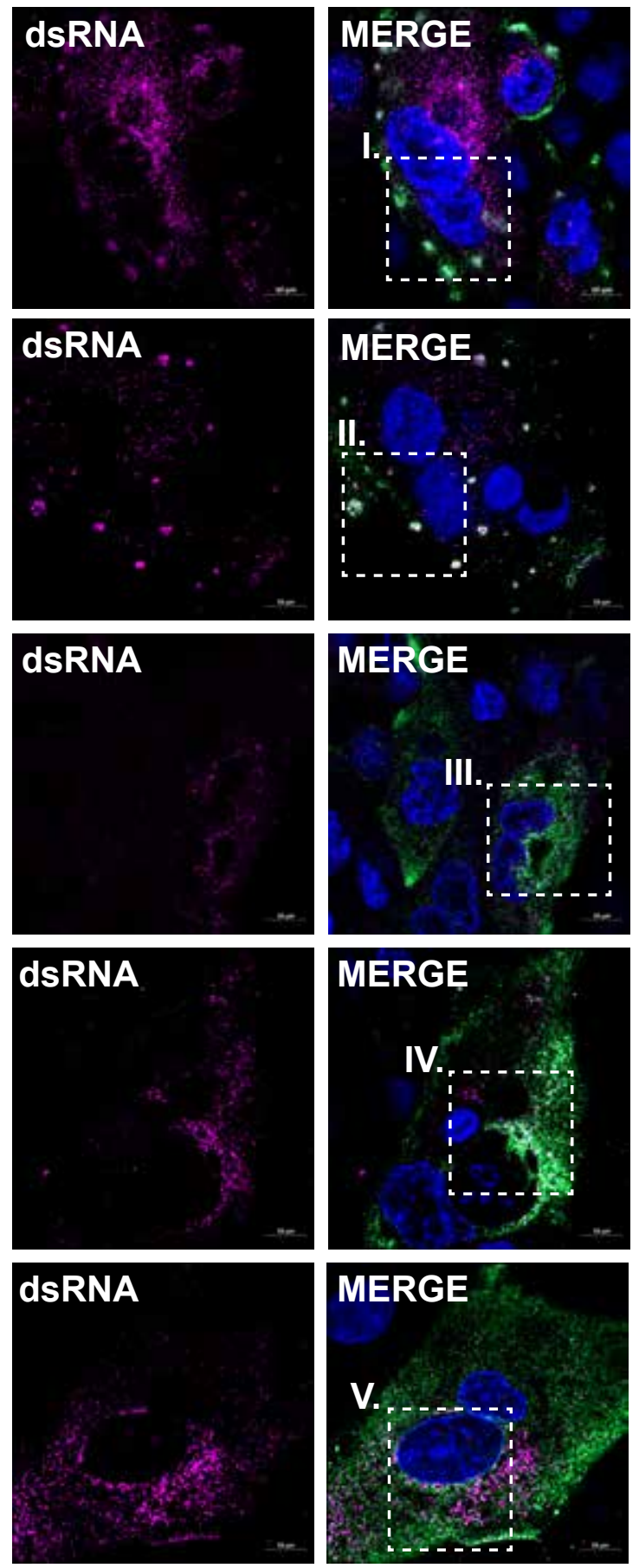

dsRNA
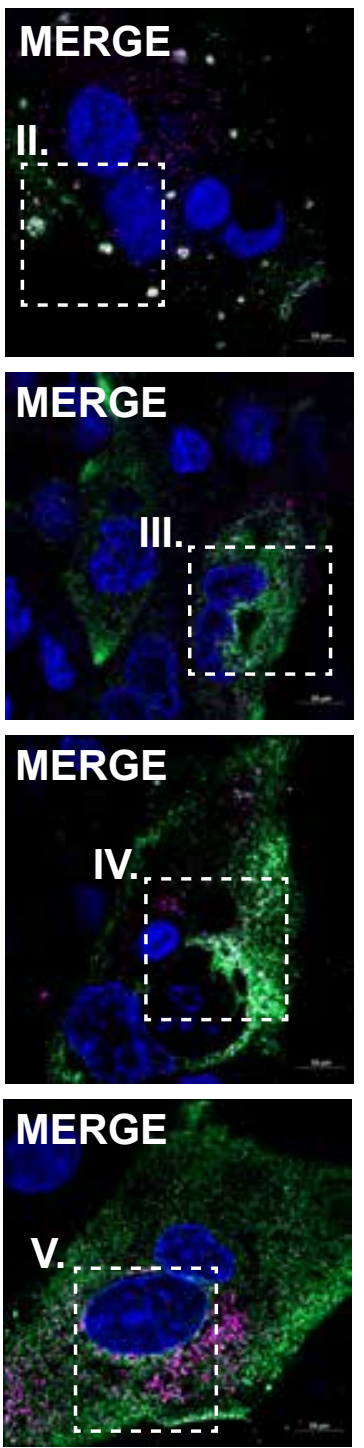

\section{MERGE}

V.

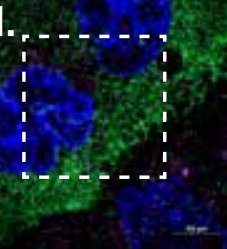

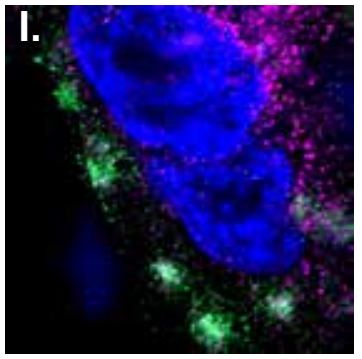
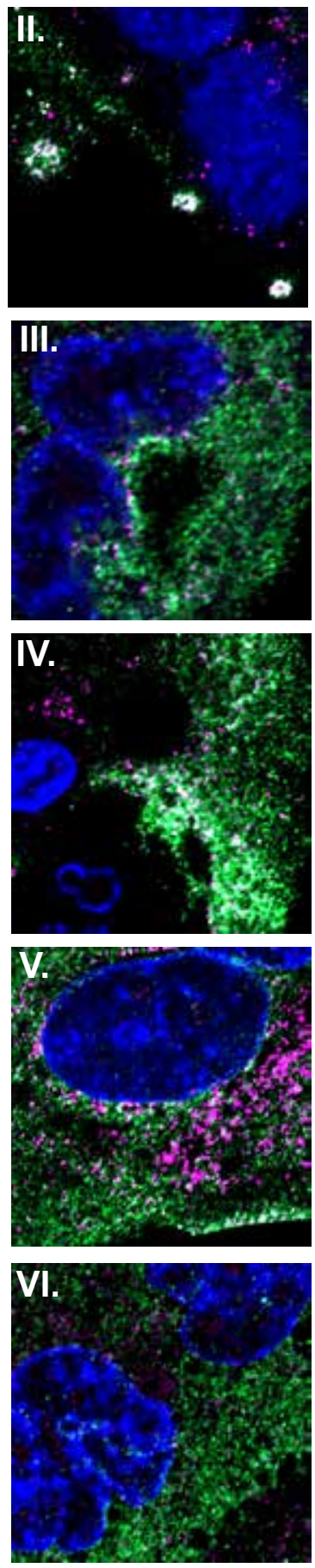

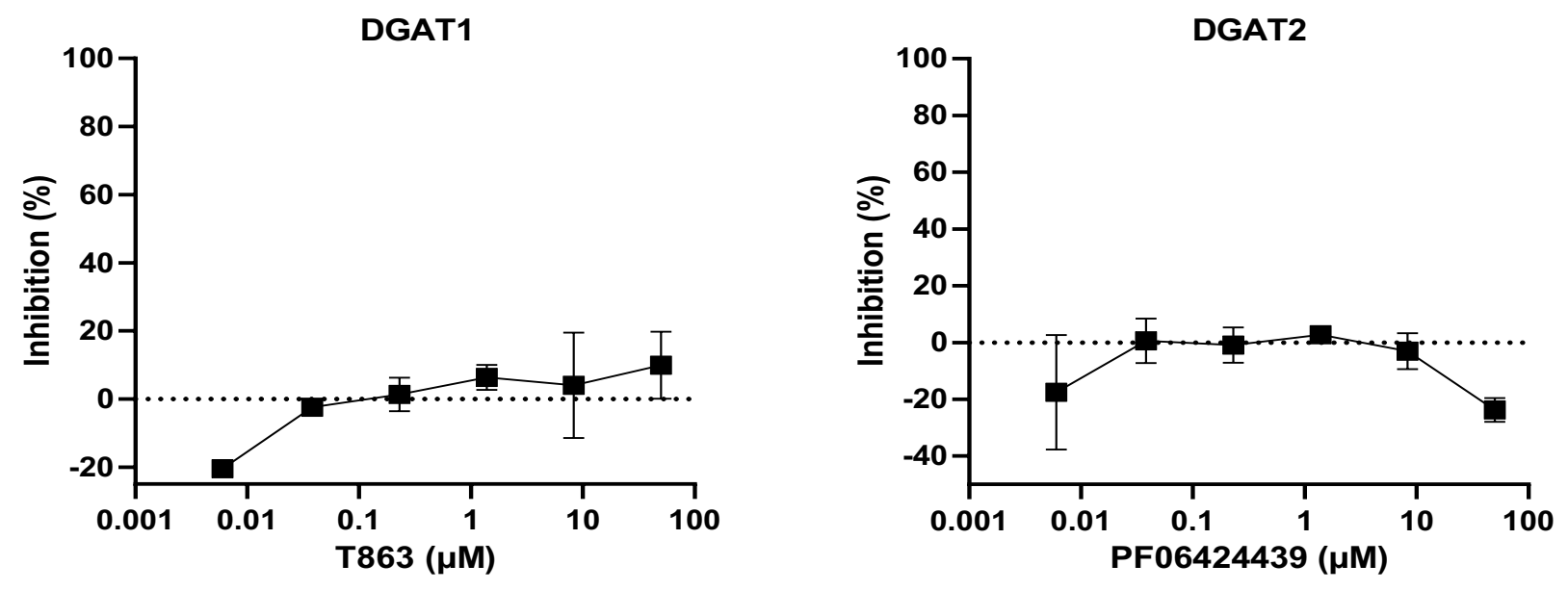

Supplemental Figure 1. 

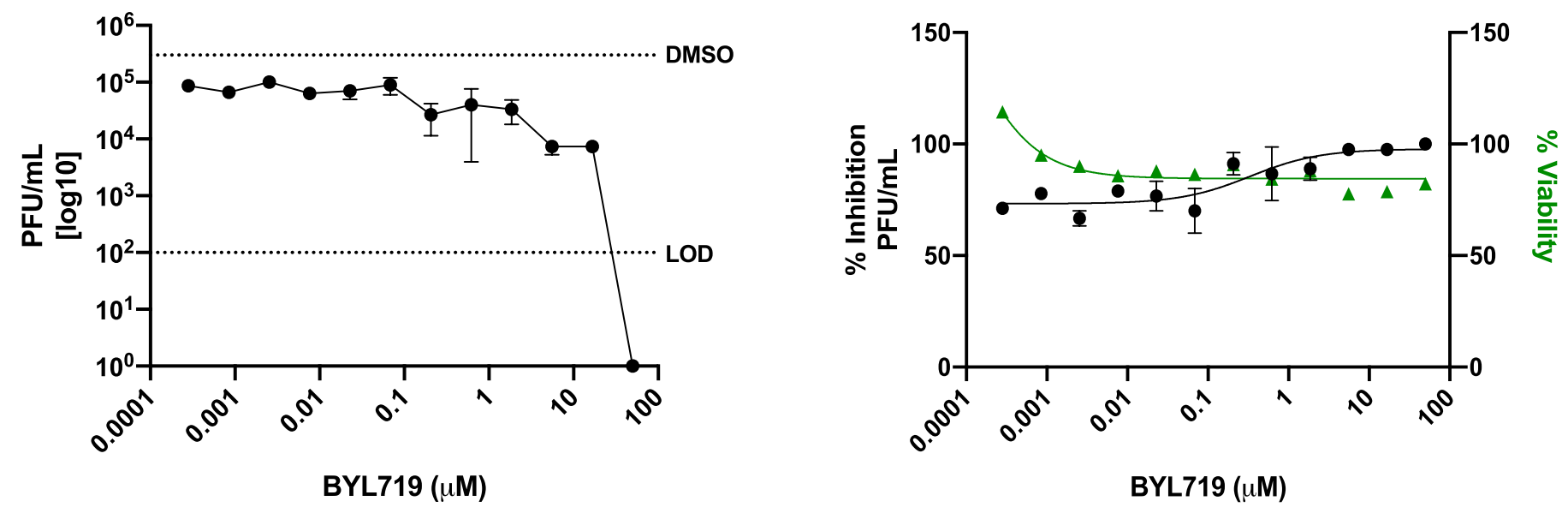

Supplemental Figure 2. 
A.
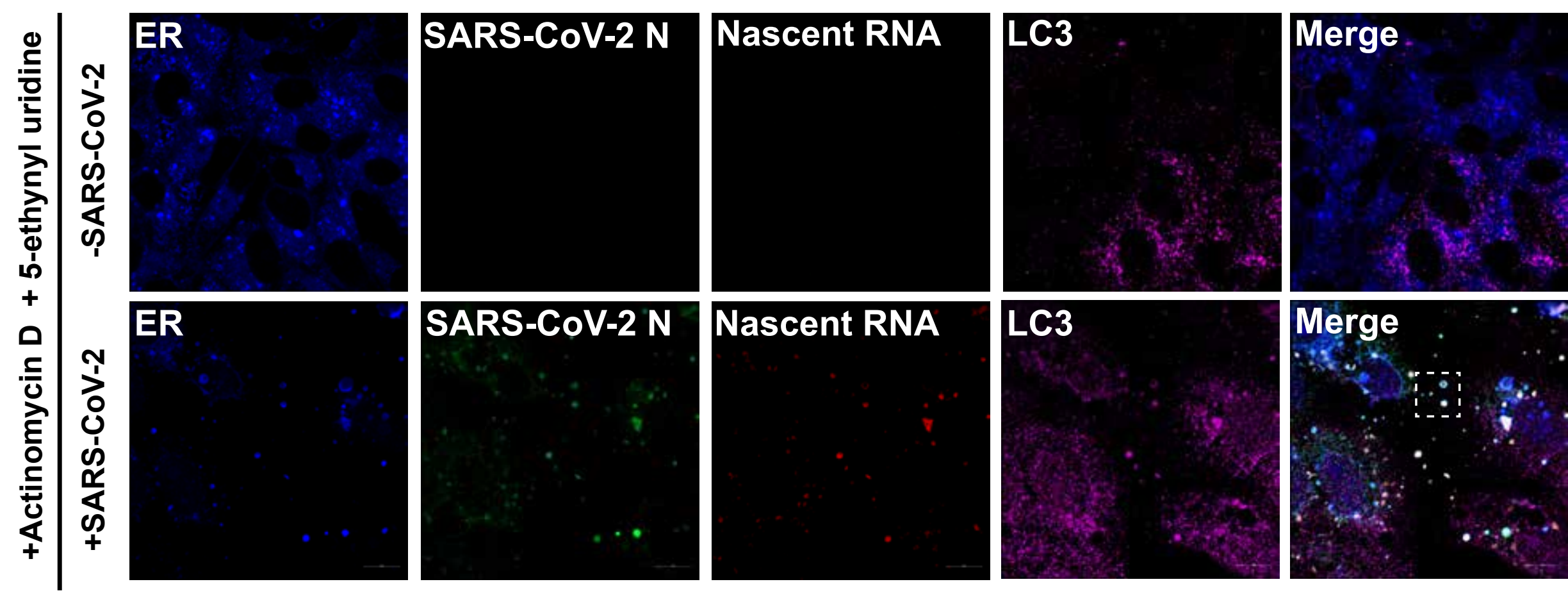

Inset

B.

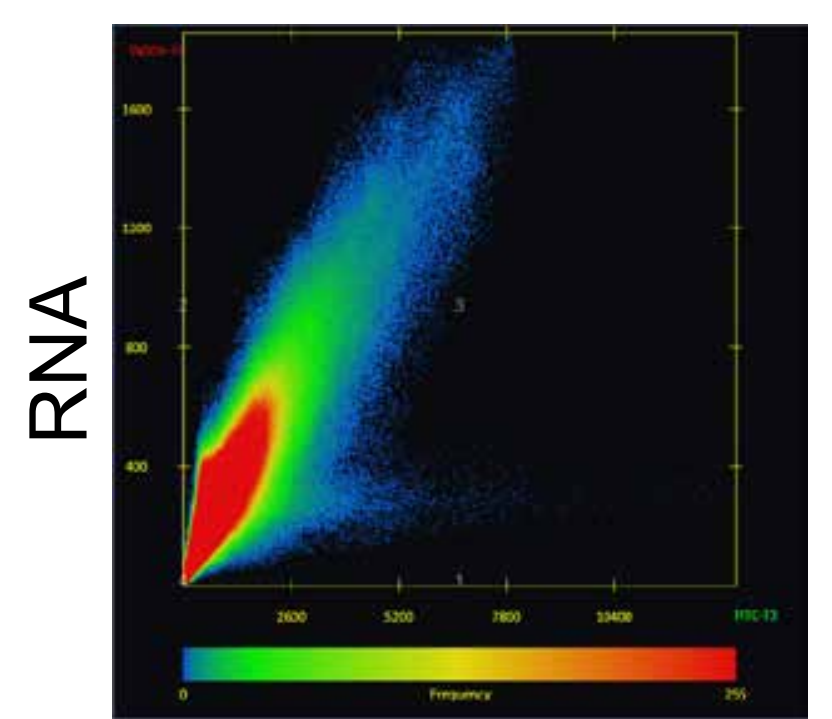

SARS-CoV-2 N

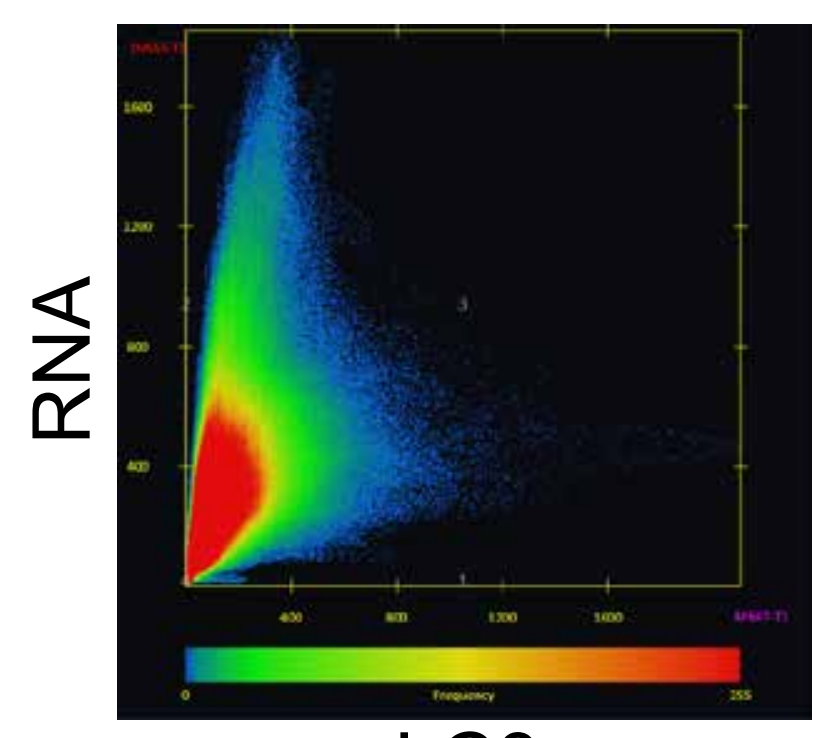

LC3 